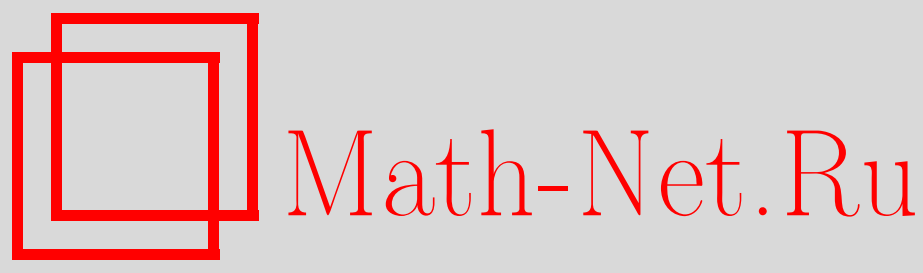

Г. В. Григорян, Р. П. Григорян, И. В. Тютин, Об изоморфизме одно- и двумерных осцилляторных и кулоноподобных теорий, ТМФ, 2013, том 176, номер 3, 339-365

DOI: https://doi.org/10.4213/tmf8552

Использование Общероссийского математического портала Math-Net.Ru подразумевает, что вы прочитали и согласны с пользовательским соглашением http://www . mathnet.ru/rus/agreement

Параметры загрузки:

IP: 54.224 .187 .69

26 апреля 2023 г., 11:17:11

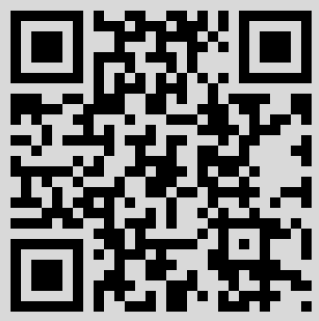




\title{
ФИЗИКА
}

Том 176, № 3

сентябрь, 2013

(C) 2013 г. $\quad$ Г. В. Григорян*, Р. П. Григорян*, И. В. Тютин ${ }^{\dagger}$

\section{ОБ ИЗОМОРФИЗМЕ ОДНО- И ДВУМЕРНЫХ ОСЦИЛЛЯТОРНЫХ И КУЛОНОПОДОБНЫХ ТЕОРИЙ}

\begin{abstract}
Представлено математически строгое описание одно- и двумерных нерелятивистских квантовых осцилляторных и кулоноподобных дуальных теорий. Произведено сравнение их спектров и полных систем (обобщенных) собственных функций самосопряженных гамильтонианов. Рассмотрены все самосопряженные операторы Шредингера этих теорий и представлены строгие решения спектральной задачи. Для построения самосопряженых расширений используется метод (асимптотических) самосопряженых граничных условий. При решении спектральной задачи используется метод направляющих функционалов Крейна. Показано, что существует изоморфизм спектров дуальных теорий (как дискретных, так и непрерывных частей спектров), а также изоморфизм соответствующих полных систем (обобщенных) собственных функций самосопряженых гамильтонианов.
\end{abstract}

Ключевые слова: дуальность, самоспряженный оператор, самосопряженное расширение.

DOI: $10.4213 / \operatorname{tmf} 8552$

\section{1. ВВЕДЕНИЕ}

Свойство уравнения Шредингера, известное как дион-осцилляторная дуальность, широко обсуждается в литературе. Типичным примером является следующий [1]: если сделать замену $r=u^{2}$ в радиальной части уравнения Шредингера $D$-мерного осциллятора $(D>2)$,

$$
\frac{d^{2} R}{d u^{2}}+\frac{D-1}{u} \frac{d R}{d u}-\frac{L(L+D-2)}{u^{2}} R+\frac{2 \mu}{\hbar^{2}}\left(E-\frac{\mu \Omega^{2} u^{2}}{2}\right) R=0
$$

(здесь $R$ - радиальная волновая функция $D$-мерного осциллятора, $L$ - собственное значение полного углового момента, $L=0,1,2, \ldots)$, то уравнение (1) преобразуется в

$$
\frac{d^{2} R}{d r^{2}}+\frac{d-1}{r} \frac{d R}{d r}-\frac{l(l+d-2)}{r^{2}} R+\frac{2 \mu}{\hbar^{2}}\left(\mathcal{E}+\frac{\alpha}{r}\right) R=0,
$$

${ }^{*}$ Национальная лаборатория им. А. Алиханяна, Ереван, Армения. E-mail: gagri@yerphi.am, rogri@yerphi.am

${ }^{\dagger}$ Федеральное государственное бюджетное учреждение науки Физический институт им. П. Н. Лебедева РАН, Москва, Россия. E-mail: tyutin@lpi.ru 
где $d=D / 2+1, l=L / 2, \mathcal{E}=-\mu \Omega^{2} / 8, \alpha=E / 4$, которое формально совпадает с уравнением Шредингера для радиальной волновой функции $d$-мерного атома водорода.

Уравнения (1) и (2) дуальны друг другу и связаны дуальным преобразованием $r=u^{2}$. Для дискретных спектров и некоторых граничных условий было доказано, что каждому состоянию уравнения (1) соответствует состояние уравнения (2) и наоборот [2], [3].

В настоящее время известен широкий класс дуальных квантово-механических моделей (см. работы [4]-[6] и ссылки в них), которые связаны заменой переменных и волновых функций в уравнении Шредингера. Однако в общем случае задача о соответствии состояний для дискретных, а также непрерывных спектров при всех значениях параметров теории и для всех самосопряженных (с.с.) гамильтонианов не рассматривалась.

В настоящей статье мы рассматриваем задачу о кулон-осцилляторной дуальности в полном объеме для уравнений Шредингера на действительной оси и на плоскости.

\section{2. УРАВНЕНИЕ ШРЕДИНГЕРА НА ДЕЙСТВИТЕЛЬНОЙ ОСИ}

\section{1. Общие замечания.}

2.1.1. Дифференциальные операции. В случае осцилляторной системы уравнение Шредингера имеет вид

$$
\check{H}_{\mathrm{O}} \Phi(u)=E_{\mathrm{O}} \Phi(u), \quad \check{H}_{\mathrm{O}}=-\partial_{u}^{2}+\lambda u^{2}, \quad u \in \mathbb{R} \backslash\{0\},
$$

где $\hbar^{2} E_{\mathrm{O}} / 2 m-$ энергия, $\hbar^{2} \lambda / 2 m-$ константа связи.

Для кулоноподобных систем уравнение Шредингера имеет вид

$$
\check{H}_{\mathrm{C}} \Psi(x)=E_{\mathrm{C}} \Psi(x), \quad \check{H}_{\mathrm{C}}=-\partial_{x}^{2}+\frac{g}{|x|}-\frac{3}{16 x^{2}}, \quad x \in \mathbb{R} \backslash\{0\},
$$

где $\hbar^{2} E_{\mathrm{C}} / 2 m$ - энергия, $\hbar^{2} g / 2 m$ - константа связи.

Заметим, что $\check{H}_{\mathrm{O}}$ и $\check{H}_{\mathrm{C}}$ представляют собой дифференциальные операции, они не являются операторами в гильбертовом пространстве $L^{2}(\mathbb{R})$ и задают только правила действия связанных с ними операторов. Для задания оператора необходимо определить набор функций (область определения, в дальнейшем просто область), на которые данные операторы действуют.

Уравнения (3) и (4) преобразуются друг в друга преобразованием дуальности

$$
x=k_{0} u|u|, \quad E_{\mathrm{C}}=-\frac{\lambda}{4 k_{0}^{2}}, \quad g=-\frac{E_{\mathrm{O}}}{4 k_{0}^{2}}, \quad \Psi(x)=|u|^{1 / 2} \Phi(u) .
$$

Далее мы установим взаимно однозначное соответствие между спектрами и полными системами собственных функций с. с. гамильтонианов в этих теориях при всех значениях параметров.

2.1.2. С. с. операторы, сохраняющие четность. Пусть $\widehat{P}$ - оператор четности, который действует на функции $\psi(y)$ из пространства $L^{2}(\mathbb{R})$ (далее $y=u$ для осцилляторной задачи и $y=x$ для кулоноподобной задачи) по правилу $\widehat{P} \psi(y)=\psi(-y)$. 
Гильбертово пространство $L^{2}(\mathbb{R})$ можно разложить в прямую ортогональную сумму $L^{2}(\mathbb{R})=L_{\mathrm{s}}^{2}(\mathbb{R}) \oplus L_{\mathrm{a}}^{2}(\mathbb{R})$ подпространства $L_{\mathrm{s}}^{2}(\mathbb{R})$ симметричных функций и подпространства $L_{\mathrm{a}}^{2}(\mathbb{R})$ антисимметричных функций: для любой функции $\psi \in L^{2}(\mathbb{R})$

$$
\psi=\psi_{\mathrm{s}}+\psi_{\mathrm{a}}, \quad \psi_{\mathrm{s}} \in L_{\mathrm{s}}^{2}(\mathbb{R}), \quad \psi_{\mathrm{a}} \in L_{\mathrm{a}}^{2}(\mathbb{R}), \quad \widehat{P} \psi_{\mathrm{s}}=\psi_{\mathrm{s}}, \quad \widehat{P} \psi_{\mathrm{a}}=-\psi_{\mathrm{a}}
$$

Легко видеть, что дифференциальные операции $\check{H}_{\mathrm{O}}$ и $\check{H}_{\mathrm{C}}$ коммутируют с $\widehat{P}$.

Ниже мы ограничимся рассмотрением только с. с. гамильтонианов $\widehat{H}_{\mathfrak{e}}$ (в дальнейшем $\widehat{H}_{\mathfrak{e}}=\widehat{H}_{\mathrm{Oe}}$ для осцилляторной задачи и $\widehat{H}_{\mathfrak{e}}=\widehat{H}_{\mathrm{Ce}}$ для кулоноподобной задачи), сохраняющих четность: $\left[\widehat{P}, \widehat{H}_{\mathfrak{e}}\right]=0$, что означает

$$
\widehat{H}_{\mathfrak{e}}=\widehat{H}_{\mathrm{se}} \oplus \widehat{H}_{\mathrm{a} \mathfrak{e}},
$$

где оператор $\widehat{H}_{\mathrm{se}}$ (оператор $\widehat{H}_{\mathrm{ae}}$ ) является с. с. частью оператора $\widehat{H}_{\mathfrak{e}}$, действующей в подпространстве $L_{\mathrm{s}}^{2}(\mathbb{R})$ (подпространстве $L_{\mathrm{a}}^{2}(\mathbb{R})$ соответственно). Наоборот, если оператор $\widehat{H}_{\mathrm{se}}$ (оператор $\left.\widehat{H}_{\mathrm{ae}}\right)$ является с. с. оператором в подпространстве $L_{\mathrm{s}}^{2}(\mathbb{R})$ (в подпространстве $L_{\mathrm{a}}^{2}(\mathbb{R})$ соответственно), то $\widehat{H}_{\mathfrak{e}}=\widehat{H}_{s \mathfrak{e}} \oplus \widehat{H}_{a \mathfrak{e}}$ является с. с. оператором в $L^{2}(\mathbb{R})$, который коммутирует с $\widehat{P}$. Таким образом, для нахождения всех с. с. операторов $\widehat{H}_{\mathfrak{e}}$, коммутирующих с $\widehat{P}$, достаточно описать все с. с. операторы $\widehat{H}_{\mathrm{se}}$ и $\widehat{H}_{\mathrm{ae}}$ в подпространствах $L_{\mathrm{s}}^{2}(\mathbb{R})$ и $L_{\mathrm{a}}^{2}(\mathbb{R})$.

Рассмотрим изометрию $T: \psi_{\mathrm{s}} \in L_{\mathrm{s}}^{2}(\mathbb{R}) \stackrel{T}{\longrightarrow} \sqrt{2} \psi\left(T: \psi_{\mathrm{a}} \in L_{\mathrm{a}}^{2}(\mathbb{R}) \stackrel{T}{\longrightarrow} \sqrt{2} \psi\right)$, где $\psi \in L^{2}\left(\mathbb{R}_{+}\right)$. Можно показать, что эта изометрия порождает взаимно однозначное соответствие $T$ между множеством с. с. операторов $\widehat{H}_{\mathrm{se}}$ (с. с. операторов $\widehat{H}_{\mathrm{ae}}$ соответственно) и множеством с. с. операторов $\hat{h}_{\mathfrak{e}}$, ассоциированных с дифференциальной

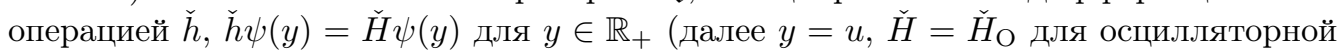
задачи и $y=x, \check{H}=\check{H}_{\mathrm{C}}$ для кулоноподобной задачи). Множество с. с. операторов $\hat{h}_{\mathfrak{e}}$ образует семейство $\hat{h}_{\zeta \mathfrak{e}}$, параметризованное величиной $\zeta,-\pi / 2 \leqslant \zeta \leqslant \pi / 2$, $\zeta=-\pi / 2 \sim \zeta=\pi / 2$ (см. ниже). Далее мы будем опускать индекс $\mathfrak{e}$ в обозначениях $\widehat{H}_{\mathrm{se}}, \widehat{H}_{\text {аe }}$ и $\hat{h}_{\zeta \mathfrak{e}}$, так что $\widehat{H}_{\zeta_{\mathrm{s}}}, \widehat{H}_{\zeta_{\mathrm{a}}} \stackrel{T}{\Longleftrightarrow} \hat{h}_{\zeta}$ и $\zeta=\zeta_{\mathrm{s}}, \zeta_{\mathrm{a}}$. Поэтому спектральный анализ с. с. оператора $\widehat{H}_{\zeta_{\mathrm{s}}}$ (оператора $\left.\widehat{H}_{\zeta_{\mathrm{a}}}\right)$ в $L_{\mathrm{s}}^{2}(\mathbb{R})\left(\right.$ в $L_{\mathrm{a}}^{2}(\mathbb{R})$ соответственно) сводится к спектральному анализу с. с. оператора $\hat{h}_{\zeta}, \zeta=\zeta_{\mathrm{s}}\left(\right.$ соответственно $\left.\zeta=\zeta_{\mathrm{a}}\right)$, в $L^{2}\left(\mathbb{R}_{+}\right)$. Имеем

$$
\operatorname{spec} \widehat{H}_{\zeta_{\mathrm{s}}}=\operatorname{spec} \hat{h}_{\zeta}, \quad \zeta=\zeta_{\mathrm{s}}, \quad \operatorname{spec} \widehat{H}_{\zeta_{\mathrm{a}}}=\operatorname{spec} \hat{h}_{\zeta}, \quad \zeta=\zeta_{\mathrm{a}},
$$

и собственные функции $Y_{\widehat{H}_{\zeta_{\mathrm{s}}}}(y)$ (собственные функции $Y_{\widehat{H}_{\zeta_{\mathrm{a}}}}(y)$ ) образуют полную систему обобщенных собственных функций оператора $\widehat{H}_{\zeta_{\mathrm{s}}}\left(\right.$ оператора $\widehat{H}_{\zeta_{\mathrm{a}}}$ ), равных

$$
\begin{aligned}
& Y_{H_{\zeta_{\mathrm{s}}}}(y)=\frac{1}{\sqrt{2}} Y_{h_{\zeta}}(|y|), \quad \zeta=\zeta_{\mathrm{s}}, \\
& Y_{H_{\zeta_{\mathrm{a}}}}(y)=\frac{1}{\sqrt{2}} \frac{y}{|y|} Y_{h_{\zeta}}(|y|), \quad \zeta=\zeta_{\mathrm{a}},
\end{aligned}
$$

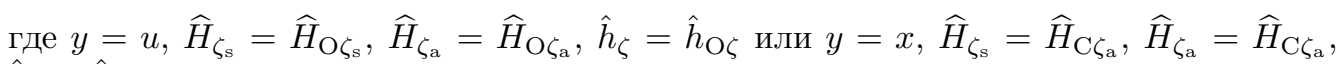
$\hat{h}_{\zeta}=\hat{h}_{\mathrm{C} \zeta}$. Далее мы приведем анализ осцилляторных и кулоноподобных систем на полуоси $\mathbb{R}_{+}$и выявим соответствие спектров и полных систем (обобщенных) собственных функций. 
Решение спектральной задачи для этих систем было рассмотрено в работе [7] (см. также [8]-[10]). Мы представим полученные в указанных работах результаты в форме, которая позволит сравнить спектры и собственные функции этих дуальных теорий. В п. 2.2 рассматривается уравнение для осциллятора, в п. 2.3 - уравнение для аниона (кулоноподобной системы). Сравнивая результаты, мы покажем, что имеется взаимно однозначное соответствие между спектрами и между (обобщенными) собственными функциями гамильтонианов для этих двух задач (см. п. 2.4).

2.1.3. С. с гамильтониан $\hat{h}_{\zeta}$. Заметим, что в обсуждаемом случае дифференциальных операций область $D_{h_{\mathfrak{e}}}$ с. с. оператора $\hat{h}_{\mathfrak{e}}$ является подпространством так называемой натуральной области

$$
D_{\breve{h}}^{*}\left(\mathbb{R}_{+}\right)=\left\{\psi_{*}: \psi_{*}, \psi_{*}^{\prime} \text { а. н. в } \mathbb{R}_{+} \backslash\{0\} ; \psi_{*}, \check{h} \psi_{*} \in L^{2}\left(\mathbb{R}_{+}\right)\right\},
$$

где а.н. обозначает, что функция абсолютно непрерывна. Можно показать, что функции $\psi_{*} \in D_{\breve{h}}^{*}\left(\mathbb{R}_{+}\right)$имеют следующее асимптотическое поведение:

для обеих систем, осцилляторной и кулоноподобной,

$$
\overline{\psi_{*}(y)} \psi_{*}^{\prime}(y)-\overline{\psi_{*}^{\prime}(y)} \psi_{*}(y) \rightarrow 0 \quad \text { при } \quad y \rightarrow \infty ;
$$

для осцилляторной системы при $u \rightarrow 0$

$$
\psi_{\mathrm{O} *}(u)=a_{1} \kappa_{0} u+a_{2}+O\left(u^{3 / 2}\right), \quad \psi_{\mathrm{O} *}^{\prime}(u)=a_{1} \kappa_{0}+O\left(u^{1 / 2}\right) ;
$$

для кулоноподобной системы при $x \rightarrow 0$

$$
\begin{aligned}
& \psi_{\mathrm{C} *}(x)=\sqrt{2} a_{1}\left(\kappa_{0} x\right)^{3 / 4}+\sqrt{2} a_{2} c_{2, \text { as }}(x)+O\left(x^{3 / 2}\right), \\
& \psi_{\mathrm{C} *}^{\prime}(x)=\frac{3 \sqrt{2} \kappa_{0} a_{1}}{4}\left(\kappa_{0} x\right)^{-1 / 4}+\sqrt{2} a_{2} c_{2, \text { as }}^{\prime}(x)+O\left(x^{1 / 2}\right),
\end{aligned}
$$

где $c_{2, \text { as }}(x)=\left(\kappa_{0} x\right)^{1 / 4}+2 g \kappa_{0} x^{5 / 4}$. Здесь $\kappa_{0}-$ некоторый фиксированный параметр, имеющий размерность обратной длины.

Область $D_{h_{\mathfrak{e}}}$ включает функции $\psi \in D_{\breve{h}}^{*}\left(\mathbb{R}_{+}\right)$, которые удовлетворяют дополнительному условию

$$
\begin{aligned}
\int_{0}^{\infty}[\overline{\psi(y)} \check{h} \psi(y)-\overline{\breve{h} \psi(y)} \psi(y)] d y & =\left.\left[\overline{\psi_{*}(y)} \psi_{*}^{\prime}(y)-\overline{\psi_{*}^{\prime}(y)} \psi_{*}(y)\right]\right|_{y \rightarrow 0}= \\
& =\kappa_{0}\left(\bar{a}_{2} a_{1}-\bar{a}_{1} a_{2}\right)=0 .
\end{aligned}
$$

Из этого соотношения следует, что существует семейство с. с. операторов $\hat{h}_{\zeta}$, где $-\pi / 2 \leqslant \zeta \leqslant \pi / 2, \zeta=-\pi / 2 \sim \zeta=\pi / 2$, областями $D_{h_{\zeta}}$ которых являются множества функций $\psi \in D_{\breve{h}}^{*}\left(\mathbb{R}_{+}\right)$, удовлетворяющих следующим условиям: для осцилляторной системы при $u \rightarrow 0$

$$
\psi_{\mathrm{O} *}(u)=a\left[\kappa_{0} u \sin \zeta+\cos \zeta\right]+O\left(u^{3 / 2}\right), \quad \psi_{\mathrm{O} *}^{\prime}(u)=a \kappa_{0} \sin \zeta+O\left(u^{1 / 2}\right) ;
$$

для кулоноподобной системы при $x \rightarrow 0$

$$
\begin{aligned}
& \psi_{\mathrm{C} *}(x)=a\left[\left(\kappa_{0} x\right)^{3 / 4} \sin \zeta+c_{2, \text { as }}(x) \cos \zeta\right]+O\left(x^{3 / 2}\right), \\
& \psi_{\mathrm{C} *}^{\prime}(x)=a\left[\left(\kappa_{0} x\right)^{3 / 4} \sin \zeta+c_{2, \text { as }}(x) \cos \zeta\right]^{\prime}+O\left(x^{1 / 2}\right) .
\end{aligned}
$$

Можно доказать, что спектр любого оператора $\hat{h}_{\zeta}$ простой (невырожденный). 
2.2. Одномерный квантовый осциллятор. В данном пункте мы рассмотрим уравнение (3). Решение спектральной задачи для этой системы было рассмотрено в работе [7] (см. также [8]).

Удобно ввести обозначение

$$
\varkappa=\left\{\begin{array}{ll}
\lambda^{1 / 4}, & \lambda \geqslant 0, \\
e^{-i \pi / 4}|\lambda|^{1 / 4}, & \lambda<0,
\end{array} \quad \varkappa^{2}= \begin{cases}\lambda^{1 / 2}, & \lambda \geqslant 0, \\
e^{-i \pi / 2}|\lambda|^{1 / 2}, & \lambda<0 .\end{cases}\right.
$$

Введем также обозначения

$$
\begin{gathered}
\rho=\rho\left(u ; \lambda, E_{\mathrm{O}}\right)=(\varkappa u)^{2}, \\
w_{\mathrm{O}}=w_{\mathrm{O}}\left(\lambda, E_{\mathrm{O}}\right)=\frac{E_{\mathrm{O}}}{4 \varkappa^{2}}, \quad \alpha_{\mathrm{O}}=\alpha_{\mathrm{O}}\left(\lambda, E_{\mathrm{O}}\right)=\frac{1}{4}-w_{\mathrm{O}}\left(\lambda, E_{\mathrm{O}}\right)
\end{gathered}
$$

и функции

$$
\begin{aligned}
& O_{\zeta}\left(u ; \lambda, E_{\mathrm{O}}\right)=O_{1}\left(u ; \lambda, E_{\mathrm{O}}\right) \sin \zeta+O_{2}\left(u ; \lambda, E_{\mathrm{O}}\right) \cos \zeta \\
& O_{1}\left(u ; \lambda, E_{\mathrm{O}}\right)=\kappa_{0} u e^{-\rho / 2} \Phi\left(\alpha_{\mathrm{O}}\left(\lambda, E_{\mathrm{O}}\right)+\frac{1}{2}, \frac{3}{2} ; \rho\right) \\
& O_{2}\left(u ; \lambda, E_{\mathrm{O}}\right)=e^{-\rho / 2} \Phi\left(\alpha_{\mathrm{O}}\left(\lambda, E_{\mathrm{O}}\right), \frac{1}{2} ; \rho\right)
\end{aligned}
$$

при $u \rightarrow 0$ имеем

$$
\begin{gathered}
O_{1}\left(u ; \lambda, E_{\mathrm{O}}\right)=\kappa_{0} u+O\left(u^{3}\right), \quad O_{2}\left(u ; \lambda, E_{\mathrm{O}}\right)=1+O\left(u^{2}\right), \\
O_{\zeta}\left(u ; \lambda, E_{\mathrm{O}}\right)=\kappa_{0} u \sin \zeta+\cos \zeta+O\left(u^{2}\right) .
\end{gathered}
$$

Функции $O_{1}\left(u ; \lambda, E_{\mathrm{O}}\right)$ и $O_{2}\left(u ; \lambda, E_{\mathrm{O}}\right)$ являются решениями уравнения $(3)$, функция $O_{\zeta}\left(u ; \lambda, E_{\mathrm{O}}\right)$ является решением уравнения $(3)$, удовлетворяющим граничным условиям (6).

2.2.1. Спектр: случай $\lambda>0$. Для любого фиксированного $\zeta$ спектр оператора $\hat{h}_{\mathrm{O} \zeta}$ дискретный,

$$
\operatorname{spec} \hat{h}_{\mathrm{O} \zeta}=\left\{E_{\mathrm{O}, n}(\lambda, \zeta), n \in \mathbb{Z}_{+}\right\},
$$

где $E_{\mathrm{O}, n}(\lambda, \zeta)$ являются решениями уравнения (см. обозначения (8))

$$
\begin{gathered}
f_{1, \mathrm{O} \zeta}\left(\lambda, E_{\mathrm{O}}\right)=0, \\
f_{1, \mathrm{O} \zeta}\left(\lambda, E_{\mathrm{O}}\right)=\frac{2 \lambda^{1 / 4}}{\kappa_{0}} \frac{\Gamma\left(\alpha_{\mathrm{O}}\left(\lambda, E_{\mathrm{O}}\right)+1 / 2\right)}{\Gamma\left(\alpha_{\mathrm{O}}\left(\lambda, E_{\mathrm{O}}\right)\right)}+\operatorname{tg} \zeta .
\end{gathered}
$$

Функция $f_{1, \mathrm{O} \zeta}(\lambda, E)$ обладает следующими свойствами:

$$
\begin{gathered}
\left.f_{1, \text { О } \zeta}(\lambda, E) \rightarrow \infty \text { при } E \rightarrow-\infty, \quad \partial_{E} f_{1, \text { о } \zeta}(\lambda, E)\right)<0, \\
\left.f_{1, \mathrm{O} \zeta}(\lambda, E)\right|_{E=E_{\mathrm{O}, n}(\lambda, 0)}=\operatorname{tg} \zeta,\left.\quad f_{1, \mathrm{O} \zeta}(\lambda, E)\right|_{E=\vartheta_{\mathrm{O}, n}(\lambda) \pm 0}= \pm \infty,
\end{gathered}
$$

где

$$
\begin{aligned}
\vartheta_{\mathrm{O}, n}(\lambda) & =2 \lambda^{1 / 2}\left[(2 n+1)+\frac{1}{2}\right] \\
E_{\mathrm{O}, n}(\lambda, 0) & =2 \lambda^{1 / 2}\left[2 n+\frac{1}{2}\right], \quad f_{1, \mathrm{O} 0}\left(\lambda, E_{\mathrm{O}, n}(\lambda, 0)\right)=0, \quad n \in \mathbb{Z}_{+} .
\end{aligned}
$$


Решения уравнения (9) удовлетворяют неравенствам

$$
\begin{gathered}
\partial_{\zeta} E_{\mathrm{O}, n}(\lambda, \zeta)>0, \quad \partial_{\lambda} E_{\mathrm{O}, n}(\lambda, \zeta)>0, \\
E_{\mathrm{O}, n}(\lambda, 0)<\vartheta_{\mathrm{O}, n}(\lambda)<E_{0, n+1}(\lambda, 0)<\vartheta_{\mathrm{O}, n+1}(\lambda), \quad n \in \mathbb{Z}_{+} .
\end{gathered}
$$

При фиксированном $\lambda$ в каждом интервале энергий $\left(\vartheta_{\mathrm{O}, n-1}(\lambda), \vartheta_{\mathrm{O}, n}(\lambda)\right), n \in \mathbb{Z}_{+}$, для фиксированного $\zeta \in(-\pi / 2, \pi / 2)$ существует один уровень энергии $E_{\mathrm{O}, n}(\lambda, \zeta)$, монотонно возрастающий от значения $\vartheta_{\mathrm{O}, n-1}(\lambda)+0$ через $E_{\mathrm{O}, n}(\lambda, 0)$ до $\vartheta_{\mathrm{O}, n}(\lambda)-0$, когда $\zeta$ меняется от $-\pi / 2+0$ через 0 до $\pi / 2-0$ (здесь мы положили $\left.\vartheta_{\mathrm{O},-1}(\lambda)=-\infty\right)$. Заметим, что справедливы соотношения

$$
\lim _{\zeta \rightarrow \pi / 2} E_{\mathrm{O}, n}(\lambda, \zeta)=\lim _{\zeta \rightarrow-\pi / 2} E_{\mathrm{O}, n+1}(\lambda, \zeta)=\vartheta_{\mathrm{O}, n}(\lambda)
$$

которые показывают эквивалентность расширений при $\zeta=-\pi / 2$ и $\zeta=\pi / 2$.

Функции $E_{\mathrm{O}, n}(\lambda, \zeta)$ при фиксированном $\zeta$ как функции параметра $\lambda$ ведут себя следующим образом. Функция $E_{\mathrm{O}, n}(\lambda, \zeta)$ при $n \geqslant 1$ и любых $\zeta$ и функция $E_{\mathrm{O}, 0}(\lambda, \zeta)$ при $\zeta \in[0, \pi / 2]$ монотонно возрастают от 0 до $\infty$, когда $\lambda$ меняется от 0 до $\infty$. Функция $E_{\mathrm{O}, 0}(\lambda, \zeta)$ при $\zeta \in(-\pi / 2,0)$, когда $\lambda$ меняется от 0 до $\infty$, монотонно возрастает от значения

$$
E_{\mathrm{O}, 0}(0, \zeta) \equiv E_{\mathrm{O} \zeta}=-\kappa_{0}^{2} \operatorname{tg}^{2} \zeta
$$

до $\infty$, пересекая ось $\lambda$ (т. е. $\left.E_{\mathrm{O}, 0}=0\right)$ в точке

$$
\lambda_{\zeta}=\left[\kappa_{0} \Gamma\left(\frac{1}{4}\right) \operatorname{tg} \frac{\zeta}{2} \Gamma\left(\frac{3}{4}\right)\right]^{4} .
$$

Полный набор ортонормированных собственных функций $\left\{U_{\zeta, n}(u ; \lambda), n \in \mathbb{Z}_{+}\right\}$ задается формулами

$$
\begin{aligned}
& U_{\zeta, n}(u ; \lambda)= Q_{\mathrm{O} \zeta, n}(\lambda) O_{\zeta}\left(u ; \lambda, E_{\mathrm{O}, n}(\lambda, \zeta)\right), \\
& Q_{\mathrm{O} \zeta, n}(\lambda)=\left\{\begin{array}{lll}
\frac{2}{\kappa_{0}}\left[\frac{\lambda^{3 / 4}(2 n+1) ! !}{\sqrt{\pi}(2 n) ! !}\right]^{1 / 2}, & \zeta=\frac{\pi}{2}, & n \in \mathbb{Z}_{+}, \\
{\left[-\kappa_{0} \partial_{E} f_{1, \mathrm{O} \zeta}\left(\lambda, E_{\mathrm{O}, n}(\lambda, \zeta)\right) \cos ^{2} \zeta\right]^{-1 / 2},} & 0<|\zeta|<\frac{\pi}{2}, & n \in \mathbb{Z}_{+}, \\
{\left[\frac{2(2 n-1) ! ! \lambda^{1 / 4}}{\sqrt{\pi}(2 n) ! !}\right]^{1 / 2},} & \zeta=0, & n \in \mathbb{Z}_{+}, \\
\frac{2}{\kappa_{0}}\left[\frac{\lambda^{3 / 4}(2 n-1) ! !}{\sqrt{\pi}(2 n-2) ! !}\right]^{1 / 2}, & \zeta=-\frac{\pi}{2}, & n \in \mathbb{N} .
\end{array}\right.
\end{aligned}
$$

2.2.2. Спектр: случай $\lambda=0$. В данном случае имеем

$$
\operatorname{spec} \hat{h}_{\mathrm{O} \zeta}= \begin{cases}\mathbb{R}_{+}, & \zeta \in[0, \pi / 2), \quad \zeta= \pm \pi / 2, \\ \mathbb{R}_{+} \cup\left\{E_{\mathrm{O} \zeta}\right\}, & \zeta \in(-\pi / 2,0),\end{cases}
$$

где мы положили $E_{\mathrm{O}, 0}(0, \zeta)=E_{0 \zeta}$. Полная система ортонормированных (обобщенных) собственных функций гамильтониана $\hat{h}_{\mathrm{O} \zeta}$ задается как

$$
\left\{U_{\zeta, E_{\mathrm{O}}}(u ; 0), E_{\mathrm{O}} \geqslant 0\right\} \quad \text { при } \quad \zeta \in[0, \pi / 2), \zeta= \pm \pi / 2
$$


и $\mathrm{KaK}$

$$
\left\{U_{\zeta, E_{\mathrm{O}}}(u ; 0), E_{\mathrm{O}} \geqslant 0 ; U_{\zeta, 0}(u ; 0)\right\} \quad \text { при } \quad \zeta \in(-\pi / 2,0),
$$

где волновые функции непрерывного спектра $U_{\zeta, E_{\mathrm{O}}}(u ; 0)$ и дискретного уровня $U_{\zeta, 0}(u ; 0)$ определены следующим образом:

$$
\begin{aligned}
U_{\zeta, E_{\mathrm{O}}}(u ; 0) & =\rho_{\mathrm{O} \zeta}\left(0, E_{\mathrm{O}}\right) O_{\zeta}\left(u ; 0, E_{\mathrm{O}}\right), & U_{\zeta, 0}(u ; 0) & =Q_{\mathrm{O} \zeta, 0}(0) O_{\zeta}\left(u ; 0, E_{\mathrm{O} \zeta}\right), \\
\rho_{\mathrm{O} \zeta}\left(0, E_{\mathrm{O}}\right) & =\left(\frac{1}{\pi} \frac{E_{\mathrm{O}}^{1 / 2}}{\kappa_{0}^{2} \sin ^{2} \zeta+E_{\mathrm{O}} \cos ^{2} \zeta}\right)^{1 / 2}, & Q_{\mathrm{O} \zeta, 0}(0) & =\frac{\sqrt{2 \kappa_{0} \sin \zeta}}{\cos \zeta} .
\end{aligned}
$$

2.2.3. Спектр: случай $\lambda<0$. В данном случае имеем $\operatorname{spec} \hat{h}_{\mathrm{O} \zeta}=\mathbb{R}$ для всех $\zeta$. Полная система ортонормированных (обобщенных) собственных функций гамильтониана $\hat{h}_{\mathrm{O} \zeta}$ задается как

$$
\left\{U_{\zeta, E_{\mathrm{O}}}(u ; \lambda)=\rho_{\mathrm{O} \zeta}\left(\lambda, E_{\mathrm{O}}\right) O_{\zeta}\left(u ; \lambda, E_{\mathrm{O}}\right), E_{\mathrm{O}} \in \mathbb{R}\right\},
$$

где

$$
\rho_{\mathrm{O} \zeta}^{2}\left(\lambda, E_{\mathrm{O}}\right)=\frac{1}{\kappa_{0}} \frac{4\left(|\lambda|^{1 / 4} / \kappa_{0}\right) e^{\pi \widetilde{w}_{\mathrm{O}}}|\Gamma(\alpha)|^{2}}{e^{2 \pi \widetilde{w}_{\mathrm{O}}}|\Gamma(\alpha)|^{4} \sin ^{2} \zeta+\left(e^{-\pi \widetilde{w}_{\mathrm{O}}}\left|\Gamma\left(\alpha_{\mathrm{O}}\right)\right|^{2} \sin \zeta+4 \pi\left(|\lambda|^{1 / 4} / \kappa_{0}\right) \cos \zeta\right)^{2}}
$$

и $\widetilde{w}_{\mathrm{O}}=\widetilde{w}_{\mathrm{O}}\left(\lambda, E_{\mathrm{O}}\right)=E_{\mathrm{O}} / 4|\lambda|^{1 / 2}$. Заметим, что стандартный спектр (когда волновые функции из области определения с. с. гамильтониана и их первые производные являются непрерывными в начале координат) возникает при $\zeta_{\mathrm{s}}=0, \zeta_{\mathrm{a}}= \pm \pi / 2$.

2.3. Одномерное кулоноподобное взаимодействие. В настоящем пункте мы рассматриваем уравнение (4). Эта задача является частным случаем обобщенной проблемы Кратцера, которая была решена в работе [7] (см. также работы [9], [10]). Здесь мы приведем результаты, которые нужны для сравнения спектров дуальных теорий.

Введем обозначения

$$
\begin{gathered}
z=z\left(x ; g, E_{\mathrm{C}}\right)=2 K x, \quad K=\sqrt{-E_{\mathrm{C}}}= \begin{cases}-i \sqrt{E_{\mathrm{C}}}, & E_{\mathrm{C}} \geqslant 0, \\
\sqrt{\left|E_{\mathrm{C}}\right|}, & E_{\mathrm{C}} \leqslant 0,\end{cases} \\
w_{\mathrm{C}}=w_{\mathrm{C}}\left(g, E_{\mathrm{C}}\right)=-\frac{g}{2 \sqrt{-E_{\mathrm{C}}}}, \quad \alpha_{\mathrm{C}}=\alpha_{\mathrm{C}}\left(g, E_{\mathrm{C}}\right)=\frac{1}{4}-w_{\mathrm{C}}\left(g, E_{\mathrm{C}}\right)
\end{gathered}
$$

и функции

$$
\begin{aligned}
& C_{\zeta}\left(x ; g, E_{\mathrm{C}}\right)=C_{1}\left(x ; g, E_{\mathrm{C}}\right) \sin \zeta+C_{2}\left(x ; g, E_{\mathrm{C}}\right) \cos \zeta, \\
& C_{1}\left(x ; g, E_{\mathrm{C}}\right)=\left(\kappa_{0} x\right)^{3 / 4} e^{-z / 2} \Phi\left(\alpha_{\mathrm{C}}+\frac{1}{2}, \frac{3}{2} ; z\right), \\
& C_{2}\left(x ; g, E_{\mathrm{C}}\right)=\left(\kappa_{0} x\right)^{1 / 4} e^{-z / 2} \Phi\left(\alpha_{\mathrm{C}}, \frac{1}{2} ; z\right) ;
\end{aligned}
$$

при $x \rightarrow 0$

$$
\begin{gathered}
C_{1}\left(x ; g, E_{\mathrm{C}}\right)=\left(\kappa_{0} x\right)^{3 / 4}+O\left(x^{7 / 4}\right), \quad C_{2}\left(x ; g, E_{\mathrm{C}}\right)=c_{2, \text { as }}(x)+O\left(x^{9 / 4}\right), \\
C_{\zeta}\left(x ; g, E_{\mathrm{C}}\right)=\left(\kappa_{0} x\right)^{3 / 4} \sin \zeta+c_{2, \text { as }}(x) \cos \zeta+O\left(x^{7 / 4}\right) .
\end{gathered}
$$

Функция $C_{\zeta}\left(x ; g, E_{\mathrm{C}}\right)$ является решением уравнения $(4)$, удовлетворяющим граничному условию $(7)$. 
2.3.1. Спектр: случай $g \geqslant 0, \zeta \in[0, \pi / 2)$ или $\zeta= \pm \pi / 2$. В данном случае при любом фиксированном $\zeta$ спектр оператора $\hat{h}_{\mathrm{C} \zeta}$ непрерывный, spec $\hat{h}_{\mathrm{C} \zeta}=\mathbb{R}_{+}$. Полная система ортонормированных (обобщенных) собственных функций гамильтониана $\hat{h}_{\mathrm{C} \zeta}$ задается как

$$
\left\{X_{\zeta, E_{\mathrm{C}}}(x ; g)=\rho_{\mathrm{C} \zeta}\left(g, E_{\mathrm{C}}\right) C_{\zeta}\left(x ; g, E_{\mathrm{C}}\right), E_{\mathrm{C}} \in \mathbb{R}_{+}\right\},
$$

где

$$
\begin{aligned}
\rho_{\mathrm{C} \zeta}^{2}\left(g, E_{\mathrm{C}}\right)= & \frac{1}{\kappa_{0}} \cdot 8 \sqrt{2}\left(E_{\mathrm{C}} / \kappa_{0}^{2}\right)^{1 / 4} e^{\pi \widetilde{w}_{\mathrm{O}}}\left|\Gamma\left(\alpha_{\mathrm{C}}\right)\right|^{2}\left\{e^{2 \pi \widetilde{w}_{\mathrm{C}}}\left|\Gamma\left(\alpha_{\mathrm{C}}\right)\right|^{4} \sin ^{2} \zeta+\right. \\
& \left.+\left(e^{-\pi \widetilde{w}_{\mathrm{C}}}\left|\Gamma\left(\alpha_{\mathrm{C}}\right)\right|^{2} \sin \zeta+4 \sqrt{2} \pi\left(\frac{E_{\mathrm{C}}}{\kappa_{0}}\right)^{1 / 4} \cos \zeta\right)^{2}\right\}^{-1}, \quad E_{\mathrm{C}}>0, \\
\rho_{\mathrm{C} \zeta}(g, 0)= & \lim _{E_{\mathrm{C}} \rightarrow+0} \rho_{\mathrm{C} \zeta}\left(g, E_{\mathrm{C}}\right)=0
\end{aligned}
$$

и $\widetilde{w}_{\mathrm{C}}=\widetilde{w}_{\mathrm{C}}\left(g, E_{\mathrm{C}}\right)=-g / 2 \sqrt{E_{\mathrm{C}}}$.

2.3.2. Спектр: случай $g \geqslant 0, \zeta \in(-\pi / 2,0)$. Пусть $g_{\zeta}=\left(\kappa_{0} / 4\right) \operatorname{tg}^{2} \zeta$. Рассмотрим по отдельности случаи $g>g_{\zeta}$ и $0 \leqslant g \leqslant g_{\zeta}$.

Пусть $g>g_{\zeta}$. В данном случае при любом фиксированном $\zeta$ спектр оператора $\hat{h}_{\mathrm{C} \zeta}$ непрерывный, spec $\hat{h}_{\mathrm{C \zeta}}=\mathbb{R}_{+}$, и полная система ортонормированных (обобщенных) собственных функций $\hat{h}_{\mathrm{C} \zeta}$ задается соотношениями (11), (12).

В случае $0 \leqslant g \leqslant g_{\zeta}$ при любом фиксированном $\zeta$ спектр оператора $\hat{h}_{\mathrm{C} \zeta}$ содержит непрерывную часть и, кроме того, существует один дискретный уровень:

$$
\operatorname{spec} \hat{h}_{\mathrm{C} \zeta}=\mathbb{R}_{+} \cup\left\{E_{\mathrm{C}, 0}(g, \zeta)\right\},
$$

где $E_{\mathrm{C}, 0}(g, \zeta)$ является решением уравнения

$$
\begin{gathered}
f_{1, \mathrm{C} \zeta}\left(g, E_{\mathrm{C}}\right)=0, \quad E_{\mathrm{C}} \leqslant 0, \\
f_{1, C \zeta}\left(g, E_{\mathrm{C}}\right)=2 \sqrt{\frac{2 K}{\kappa_{0}}} \frac{\Gamma\left(\alpha_{\mathrm{C}}\left(g, E_{\mathrm{C}}\right)+1 / 2\right)}{\Gamma\left(\alpha_{\mathrm{C}}\left(g, E_{\mathrm{C}}\right)\right)}+\operatorname{tg} \zeta
\end{gathered}
$$

(см. обозначения (10)). Решение $E_{\mathrm{C}, 0}(g, \zeta)$ этого уравнения имеет следующие свойства: $\partial_{\zeta} E_{\mathrm{C}, 0}(g, \zeta)>0$ и $\partial_{g} E_{\mathrm{C}, 0}(g, \zeta)>0$; при фиксированном значении $g$ функция $E_{\mathrm{C}, 0}(g, \zeta)$ монотонно возрастает от $-\infty$ до 0 , когда $\zeta$ изменяется от $-\pi / 2+0$ до $\zeta_{g}=-\operatorname{arctg}\left(4 g / \kappa_{0}\right)^{1 / 2}$; при фиксированном $\zeta$ функция $E_{\mathrm{C}, 0}(g, \zeta)$ монотонно возрастает от $E_{\mathrm{C} \zeta}$ до 0 , когда $g$ изменяется от 0 до $g_{\zeta}$, где

$$
E_{\mathrm{C} \zeta} \equiv E_{\mathrm{C}, 0}(0, \zeta)=-\left(\frac{\sqrt{\kappa_{0}} \Gamma(1 / 4) \operatorname{tg} \zeta}{2 \sqrt{2} \Gamma(3 / 4)}\right)^{4} .
$$

Полная система ортонормированных (обобщенных) собственных функций оператоpa $\hat{h}_{\mathrm{C} \zeta}$ задается как

$$
\left\{X_{\zeta, E_{\mathrm{C}}}(x ; g), E_{\mathrm{C}} \in \mathbb{R}_{+} ; X_{\zeta, 0}(x ; g)\right\},
$$

где $X_{\zeta, E_{\mathrm{C}}}(x ; g)$ определяется соотношениями $(11),(12)$ и

$$
\begin{gathered}
X_{\zeta, 0}(x ; g)=Q_{C \zeta, 0}(g) C_{\zeta}\left(x ; g, E_{\mathrm{C}, 0}(g, \zeta)\right), \\
Q_{C \zeta, 0}(g)=\sqrt{2}\left[-\kappa_{0}^{1 / 2} \partial_{E_{\mathrm{C}}} f_{1, \mathrm{C} \zeta}\left(g, E_{\mathrm{C}, 0}(g, \zeta)\right) \cos ^{2} \zeta\right]^{-1 / 2} .
\end{gathered}
$$


2.3.3. Спектр: случай $g<0$. В данном случае при любом фиксированном $\zeta$ спектр оператора $\hat{h}_{\mathrm{C} \zeta}$ содержит непрерывную часть и семейство дискретных уровней:

$$
\operatorname{spec} \hat{h}_{\mathrm{C} \zeta}=\mathbb{R}_{+} \cup\left\{E_{\mathrm{C}, n}(g, \zeta), n \in \mathbb{Z}_{+}\right\},
$$

где $E_{\mathrm{C}, n}(g, \zeta)<0$ являются решениями уравнений

$$
f_{1, \mathrm{C} \zeta}\left(g, E_{\mathrm{C} \zeta}\right)=0 .
$$

Функция $f_{1, \mathrm{C} \zeta}(g, E)$, заданная в $(13)$, имеет следующие свойства:

$$
\begin{gathered}
f_{1, \mathrm{C} \zeta}(g, E) \rightarrow \infty \text { при } E \rightarrow-\infty, \quad \partial_{E} f_{1, \mathrm{C} \zeta}(g, E)<0, \\
\left.f_{1, \mathrm{C} \zeta}(g, E)\right|_{E=\vartheta_{\mathrm{C}, n}(g) \pm 0}= \pm \infty,\left.\quad f_{1, \mathrm{C} \zeta}(g, E)\right|_{E=E_{\mathrm{C}, n}(g, 0)}=\operatorname{tg} \zeta
\end{gathered}
$$

где

$$
\begin{aligned}
\vartheta_{\mathrm{C}, n}(g) & =-g^{2}\left[(2 n+1)+\frac{1}{2}\right]^{-2}, \\
E_{\mathrm{C}, n}(g, 0) & =-g^{2}\left[2 n+\frac{1}{2}\right]^{-2}, \quad f_{1, \mathrm{C} 0}\left(g, E_{\mathrm{C}, n}(g, 0)\right)=0, \quad n \in \mathbb{Z}_{+} .
\end{aligned}
$$

Решения $E_{\mathrm{C}, n}(g, 0)$ удовлетворяют неравенствам

$$
\begin{gathered}
\partial_{\zeta} E_{\mathrm{C}, n}(g, \zeta)>0, \quad \partial_{g} E_{\mathrm{C}, n}(g, \zeta)>0, \\
E_{\mathrm{C}, n}(g, 0)<\vartheta_{\mathrm{C}, n}(g)<E_{\mathrm{C}, n+1}(g, 0)<\vartheta_{\mathrm{C}, n+1}(g), \quad n \in \mathbb{Z}_{+} .
\end{gathered}
$$

Таким образом, в каждом интервале энергий $\left(\vartheta_{\mathrm{C}, n-1}(g), \vartheta_{\mathrm{C}, n}(g)\right), n \in \mathbb{Z}_{+}$, при фиксированном $\zeta \in(-\pi / 2, \pi / 2)$ существует один уровень энергии $E_{\mathrm{C}, n}(g, \zeta)$, монотонно возрастающий от значения $\vartheta_{\mathrm{C}, n-1}(g)+0$ через $E_{\mathrm{C}, n}(g, 0)$ до $\vartheta_{\mathrm{C}, n}(g)-0$, когда $\zeta$ пробегает значения от $-\pi / 2+0$ через 0 до $\pi / 2-0$ (мы положили $\vartheta_{C-1}(g)=-\infty$ ). Заметим, что выполняются соотношения

$$
\lim _{\zeta \rightarrow \pi / 2} E_{\mathrm{C}, n}(g, \zeta)=\lim _{\zeta \rightarrow-\pi / 2} E_{\mathrm{C}, n+1}(g, \zeta)=\vartheta_{\mathrm{C}, n}(g)
$$

которые свидетельствуют об эквивалентности расширений для $\zeta=-\pi / 2$ и $\zeta=\pi / 2$.

При фиксированном $\zeta$ функции $E_{\mathrm{C}, n}(g, \zeta)$ как функции параметра $g$ ведут себя следующим образом: $E_{\mathrm{C}, n}(g, \zeta)$ при $n \geqslant 1$ и любом $\zeta$ и $E_{\mathrm{C}, 0}(g, \zeta)$ при $\zeta \in[0, \pi / 2]$ возрастают монотонно от $-\infty$ до -0 , когда $g$ изменяется от $-\infty$ до -0 ; функция $E_{\mathrm{C}, 0}(g, \zeta)$ при $\zeta \in(-\pi / 2,0)$ возрастает монотонно от $-\infty$ до $E_{\mathrm{C} \zeta}$, когда $g$ изменяется от $-\infty$ до 0 .

Полная система ортонормированных (обобщенных) собственных функций оператора $\hat{h}_{\mathrm{C} \zeta}$ задается как

$$
\left\{X_{\zeta, E_{\mathrm{C}}}(x ; g), E_{\mathrm{C}} \in \mathbb{R}_{+} ; X_{\zeta, n}(x ; g), n \in \mathbb{Z}_{+}\right\}
$$

где $X_{\zeta, E_{\mathrm{C}}}(x ; g)$ определяется соотношениями $(11),(12)$ и

$$
X_{\zeta, n}(x ; g)=Q_{C \zeta, n}(g) C_{\zeta}\left(x ; g, E_{\mathrm{C}, n}(g, \zeta)\right),
$$




$$
Q_{C \zeta, n}(g)=\left\{\begin{array}{lll}
{\left[\frac{8 \sqrt{2}\left|\vartheta_{\mathrm{C}, n}(g)\right|^{7 / 4}(2 n+1) ! !}{\sqrt{\pi} \kappa_{0}^{3 / 2}|g|(2 n) ! !}\right]^{1 / 2},} & \zeta=\frac{\pi}{2}, & n \in \mathbb{Z}_{+}, \\
\sqrt{2}\left[-\kappa_{0} \partial_{E} f_{1, \mathrm{C} \zeta}\left(g, E_{\mathrm{C}, n}(g, \zeta)\right) \cos ^{2} \zeta\right]^{-1 / 2}, & 0<\mid \zeta<\frac{\pi}{2}, & n \in \mathbb{Z}_{+}, \\
{\left[\frac{2 \sqrt{2}\left|E_{\mathrm{C}, n}(g, 0)\right|^{5 / 4}(2 n-1) ! !}{\sqrt{\pi} \kappa_{0}^{1 / 2}|g|(2 n) ! !}\right]^{1 / 2},} & \zeta=0, & n \in \mathbb{Z}_{+}, \\
{\left[\frac{8 \sqrt{2}\left|\vartheta_{\mathrm{C}, n-1}(g)\right|^{7 / 4}(2 n-1) ! !}{\sqrt{\pi} \kappa_{0}^{3 / 2}|g|(2 n-2) ! !}\right]^{1 / 2},} & \zeta=-\frac{\pi}{2}, & n \in \mathbb{N} .
\end{array}\right.
$$

Отметим, что при фиксированном значении $\zeta \in(-\pi / 2,0)$ две кривые $E_{\mathrm{C}, 0}(g, \zeta)$ при $-\infty<g<0$ и при $0 \leqslant g \leqslant g_{\zeta}$ являются в действительности двумя частями одной кривой $E_{\mathrm{C}, 0}(g, \zeta),-\infty<g \leqslant g_{\zeta}$.

Функция $E_{\mathrm{C}, 0}(g, \zeta),-\infty<g \leqslant g_{\zeta}$, при $\zeta \in(-\pi / 2,0)$ обладает следующими свойствами: $\partial_{\zeta} E_{\mathrm{C}, 0}(g, \zeta)>0$ и $\partial_{g} E_{\mathrm{C}, 0}(g, \zeta)>0$; при фиксированном $g \leqslant 0$ функция $E_{\mathrm{C}, 0}(g, \zeta)$ возрастает монотонно от $-\infty$ до $E_{\mathrm{C}, 0}(g, 0)-0$, когда $\zeta$ растет от $-\pi / 2+0$ до -0 ; при фиксированном $g>0$ функция $E_{\mathrm{C}, 0}(g, \zeta)$ возрастает монотонно от $-\infty$ до 0 , когда $\zeta$ меняется монотонно от $-\pi / 2+0$ до $\zeta_{g}$; при фиксированном $\zeta$ и изменении $g$ от $-\infty$ до $g_{\zeta}$ функция $E_{\mathrm{C}, 0}(g, \zeta)$ возрастает монотонно от $-\infty$ до 0 , пересекая ось $E_{\mathrm{C}}($ т. е. $g=0)$ в точке $E_{\mathrm{C} \zeta}$.

\section{4. Сравнение спектров теорий с осцилляторным и кулоноподобным} (одномерным анионным) потенциалами. Напомним, что в данном случае соответствие двух теорий задается преобразованиями

$$
x=\kappa_{0} u^{2}, \quad E_{\mathrm{C}}=-\frac{\lambda}{4 \kappa_{0}^{2}}, \quad g=-\frac{E_{\mathrm{O}}}{4 \kappa_{0}}, u=\sqrt{\frac{x}{\kappa_{0}}}, \quad E_{\mathrm{O}}=-4 \kappa_{0} g, \quad \lambda=-4 \kappa_{0}^{2} E_{\mathrm{C}} .
$$

В результате возникает следующее соответствие между параметрами и функциями осцилляторной и кулоноподобной систем:

$$
\begin{gathered}
K=\frac{\sqrt{\lambda}}{2 \kappa_{0}}=\frac{\varkappa^{2}}{2 \kappa_{0}}, \quad \sqrt{K}=\frac{\varkappa}{\sqrt{2 \kappa_{0}}}, \quad z=\rho, \quad \sqrt{g}=\frac{\sqrt{-E_{\mathrm{O}}}}{2 \sqrt{\kappa_{0}}} ; \\
w_{\mathrm{C}}\left(g, E_{\mathrm{C}}\right)=w_{\mathrm{O}}\left(\lambda, E_{\mathrm{O}}\right), \quad \widetilde{w}_{\mathrm{C}}\left(g, E_{\mathrm{C}}\right)=\widetilde{w}_{\mathrm{O}}\left(\lambda, E_{\mathrm{O}}\right),
\end{gathered}
$$

кроме того,

$$
\alpha_{\mathrm{C}}\left(g, E_{\mathrm{C}}\right)=\alpha_{\mathrm{O}}\left(\lambda, E_{\mathrm{O}}\right) ;
$$

$f_{1, \mathrm{C} \zeta}\left(g, E_{\mathrm{C}}\right)=f_{1, \mathrm{O} \zeta}\left(\lambda, E_{\mathrm{O}}\right), \quad f_{1, \mathrm{C} \zeta}\left(g, E_{\mathrm{C}, n}(g, \zeta)\right)=0 \Longleftrightarrow f_{1, \mathrm{O} \zeta}\left(\lambda, E_{\mathrm{O}, n}(\lambda, \zeta)\right)=0$,

$$
\begin{gathered}
E_{\mathrm{C} \zeta}=-\left[\frac{\sqrt{\kappa_{0}} \Gamma(1 / 4) \operatorname{tg} \zeta}{2 \sqrt{2} \Gamma(3 / 4)}\right]^{4}=-\frac{1}{4 \kappa_{0}^{2}}\left[\frac{\kappa_{0} \Gamma(1 / 4) \operatorname{tg} \zeta}{2 \Gamma(3 / 4)}\right]^{4}=-\frac{\lambda_{\zeta}}{4 \kappa_{0}^{2}}, \\
g_{\zeta}=\frac{\kappa_{0}}{4} \operatorname{tg}^{2} \zeta=-\frac{-\kappa_{0}^{2} \operatorname{tg}^{2} \zeta}{4 \kappa_{0}}=-\frac{E_{\mathrm{O} \zeta}}{4 \kappa_{0}} \\
\rho_{\mathrm{C} \zeta}\left(g, E_{\mathrm{C}}\right)=\sqrt{2} \rho_{\mathrm{O} \zeta}\left(\lambda, E_{\mathrm{O}}\right), \\
C_{k}\left(x ; g, E_{\mathrm{C}}\right)=\left(\kappa_{0} x\right)^{1 / 4} O_{k}\left(u ; \lambda, E_{\mathrm{O}}\right), \quad k=1,2,
\end{gathered}
$$


и для $g \in \mathbb{R}, E_{\mathrm{O}} \in \mathbb{R}$

$$
\begin{aligned}
X_{\zeta, E_{\mathrm{C}}}(x ; g) & =\sqrt{2}\left(\kappa_{0} x\right)^{1 / 4} U_{\zeta, E_{\mathrm{O}}}(u ; \lambda), \quad E_{\mathrm{C}} \geqslant 0, \quad \lambda \leqslant 0, \\
X_{\zeta, n}(x ; g) & =\frac{Q_{C \zeta, n}(g)}{Q_{\mathrm{O} \zeta, n}(\lambda)}\left(\kappa_{0} x\right)^{1 / 4} U_{\zeta, n}(u ; \lambda), \quad E_{\mathrm{C}} \leqslant 0, \quad \lambda \geqslant 0, \quad n \in \mathbb{Z}_{+},
\end{aligned}
$$

где $E_{\mathrm{C}, n}\left(g\left(E_{\mathrm{O}, n}(\lambda, \zeta)\right), \zeta\right)=-\lambda / 4 \kappa_{0}^{2}$ и $E_{\mathrm{O}, n}\left(\lambda\left(E_{\mathrm{C}, n}(g, \zeta)\right), \zeta\right)=-4 \kappa_{0} g$.

Напомним, что наборы $\left\{X_{\zeta, E_{\mathrm{C}}}(x ; g), X_{\zeta, n}(x ; g)\right\}$ и $\left\{U_{\zeta, E_{\mathrm{O}}}(u ; \lambda), U_{\zeta, n}(u ; \lambda)\right\}$ образуют полные ортонормированные системы (обобщенных) собственных функций в гильбертовых пространствах $L^{2}\left(\mathbb{R}_{+}, x\right)$ и $L^{2}\left(\mathbb{R}_{+}, u\right)$ соответственно.

На рис. 1 показаны решения соответствующих уравнений для дискретного спектра. Можно видеть полное соответствие решений.

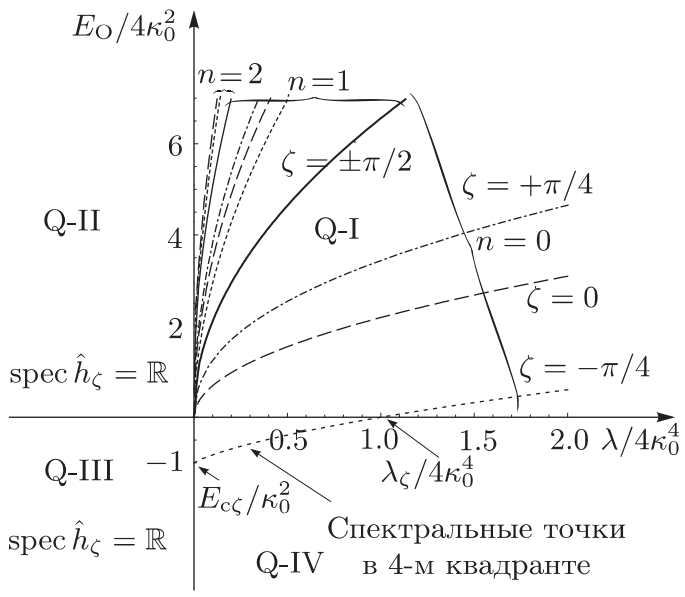

a

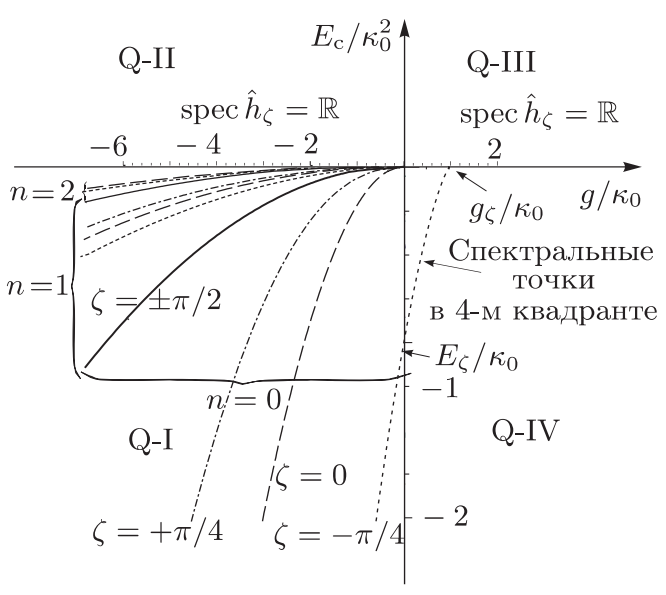

б

Рис. 1. Спектр одномерной осцилляторной (а) и одномерной кулоноподобной (б) систем. Соответствующие друг другу квадранты на обоих графиках обозначены одинаково как Q-I, Q-II, Q-III, Q-IV.

Соответствие дискретных спектров становится еще более явным, если ввести безразмерные переменные $\mathcal{W}$ и $\mathcal{V}$, которые в случае осциллятора равны

$$
\mathcal{W}=\mathcal{W}_{\mathrm{O}}=\frac{\lambda}{4 \kappa_{0}^{4}}, \quad \mathcal{V}=\mathcal{V}_{\mathrm{O}}=\frac{E_{\mathrm{O}}}{4 \kappa_{0}^{2}},
$$

а в случае кулоноподобного взаимодействия равны

$$
\mathcal{W}=\mathcal{W}_{\mathrm{C}}=-\frac{E_{\mathrm{C}}}{\kappa_{0}^{2}}, \quad \mathcal{V}=\mathcal{V}_{\mathrm{C}}=-\frac{g}{\kappa_{0}}
$$

Тогда в обоих случаях уравнения (9) и (14) в терминах этих переменных совпадают и имеют вид

$$
2 \sqrt{2} \mathcal{W}^{1 / 4} \frac{\Gamma(3 / 4-\mathcal{V} / 2 \sqrt{\mathcal{W}})}{\Gamma(1 / 4-\mathcal{V} / 2 \sqrt{\mathcal{W}})}+\operatorname{tg} \zeta=0, \quad \mathcal{W} \geqslant 0
$$




\section{3. УРАВНЕНИЕ ШРЕДИНГЕРА НА ПЛОСКОСТИ}

\section{1. Общие замечания.}

3.1.1. Дифференциальные операции. Запишем уравнения Шредингера в полярных координатах. Для осцилляторной системы уравнение Шредингера имеет вид

$$
\begin{gathered}
\check{H}_{\mathrm{O}} \Phi(u, \varphi)=E_{\mathrm{O}} \Phi(u, \varphi), \\
\check{H}_{\mathrm{O}}=-\partial_{u}^{2}-\frac{1}{u} \partial_{u}-\frac{1}{u^{2}} \partial_{\varphi}^{2}+\lambda u^{2}, \quad u \in \mathbb{R}_{+}, \quad 0 \leqslant \varphi \leqslant 2 \pi,
\end{gathered}
$$

где $\hbar^{2} E_{\mathrm{O}} / 2 m-$ энергия, $\hbar^{2} \lambda / 2 m-$ константа связи.

Для кулоноподобной системы уравнение Шредингера имеет вид

$$
\begin{gathered}
\check{H}_{\mathrm{C}} \Psi(x, \phi)=E_{\mathrm{C}} \Psi(x, \phi), \\
\check{H}_{\mathrm{C}}=-\partial_{x}^{2}-\frac{1}{x} \partial_{x}-\frac{1}{x^{2}} \partial_{\phi}^{2}+\frac{g}{x}, \quad x \in \mathbb{R}_{+}, \quad 0 \leqslant \phi \leqslant 4 \pi,
\end{gathered}
$$

где $\hbar^{2} E_{\mathrm{C}} / 2 m$ - энергия, $\hbar^{2} g / 2 m$ - константа связи.

Напомним, что $\check{H}_{\mathrm{O}}$ и $\check{H}_{\mathrm{C}}$ являются дифференциальными операциями, а не операторами в гильбертовом пространстве $L^{2}\left(\mathbb{R}^{2}\right)$. Для получения операторов необходимо определить набор функций (области), на которые действуют эти операторы. Уравнения (15) и (16) преобразуются друг в друга преобразованием дуальности

$$
x=k_{0} u^{2}, \quad \phi=2 \varphi, \quad E_{\mathrm{C}}=-\frac{\lambda}{4 k_{0}^{2}}, \quad g=-\frac{E_{\mathrm{O}}}{4 k_{0}}, \quad \Psi(x, \phi)=\left(k_{0} u\right)^{1 / 2} \Phi(u, \varphi) .
$$

Мы установим взаимно однозначное соответствие между спектрами и полными системами собственных функций с. с. гамильтонианов этих двух задач при всех значениях параметров.

3.1.2. Редукция к радиальной задаче. Пусть унитарный оператор вращения $\widehat{S}_{\alpha}$, $\widehat{S}_{\alpha} \in S O(2)$, действует на функции $\psi(y, \theta)$ (здесь $y=u, \theta=\varphi$ или $y=x, \theta=\phi$ ), принадлежащие гильбертовому пространству $\mathfrak{H}=L^{2}(\mathbb{R}, y)$, следующим образом:

$$
\widehat{S}_{\alpha} \psi(y, \theta)=\psi(y, \theta-\alpha) .
$$

Гильбертово пространство $\mathfrak{H}$ и функции $\psi \in \mathfrak{H}$ можно представить в виде

$$
\begin{array}{ccc}
\mathfrak{H}=\bigoplus_{m \in \mathbb{Z}} \mathfrak{H}_{m}, & \widehat{S}_{\alpha} \mathfrak{H}_{m}=e^{-i \sigma m \alpha} \mathfrak{H}_{m}, \\
\psi(y, \theta)=\sum_{m \in \mathbb{Z}} \psi_{m}(y, \theta), & \psi_{m}(y, \theta)=\frac{1}{\sqrt{y}} \sqrt{\frac{\sigma}{2 \pi}} e^{i \sigma m \theta} f_{m}(y),
\end{array}
$$

где $\sigma=\sigma_{\mathrm{O}}=1$ для осцилляторной задачи и $\sigma=\sigma_{\mathrm{C}}=1 / 2$ для кулоноподобной задачи. Функции $\psi_{m}(y, \theta) \in \mathfrak{H}_{m}$ являются собственными для оператора углового момента $\widehat{L}_{z}=-i \hbar \partial_{\theta}$ (с собственными значениями $\left.\sigma m\right)$, функции $f_{m}(y) \in \mathfrak{h}_{m} \equiv L^{2}\left(\mathbb{R}_{+}\right)$, где $L^{2}\left(\mathbb{R}_{+}\right)$- гильбертово пространство квадратично-интегрируемых функций на полуоси $\mathbb{R}_{+}$со скалярным произведением

$$
(f, g)=\int_{\mathbb{R}_{+}} \overline{f(y)} g(y) d y
$$


индуцированным скалярным произведением

$$
\left(\psi_{1}, \psi_{2}\right)=\int \overline{\psi_{1}(y, \theta)} \psi_{2}(y, \theta) y d y d \theta
$$

в начальном гильбертовом пространстве. Легко видеть,что дифференциальная операция $\check{H}$ коммутирует с операторами вращения $\widehat{S}_{\alpha}$.

Ниже мы ограничимся рассмотрением с. с. гамильтонианов $\widehat{H}_{\mathfrak{e}}$ (здесь $\widehat{H}_{\mathfrak{e}}=\widehat{H}_{\mathrm{Oe}}$ или $\left.\widehat{H}_{\mathfrak{e}}=\widehat{H}_{\mathrm{Ce}}\right)$, сохраняющих вращательную инвариантность: $\left[\widehat{S}_{\alpha} \widehat{H}_{\mathfrak{e}}\right]=0$, это означает, что

$$
\widehat{H}_{\mathfrak{e}}=\bigoplus_{m \in \mathbb{Z}} \widehat{H}_{\mathfrak{e} m}
$$

где операторы $\widehat{H}_{\mathfrak{e} m}$ являются с. с. частями оператора $\widehat{H}_{\mathfrak{e}}$, действующими на подпространствах $\mathfrak{H}_{m}$. И наоборот, если $\widehat{H}_{\mathfrak{e} m}-$ с. с. операторы на подпространствах $\mathfrak{H}_{m}$, то оператор $\widehat{H}_{\mathfrak{e}}=\bigoplus_{m \in \mathbb{Z}} \widehat{H}_{\mathfrak{e} m}$ является с. с. оператором в $L^{2}(\mathbb{R})$, который коммутирует с $\widehat{S}_{\alpha}$. Таким образом, достаточно описать все с. с. операторы $\widehat{H}_{\mathfrak{e} m}$ в подпространствах $\mathfrak{H}_{m}$, чтобы найти все с. с. операторы $\widehat{H}_{\mathfrak{e}}$, коммутирующие с $\widehat{S}_{\alpha}$.

C. с. оператор $\widehat{H}_{\mathfrak{e}}$ действует в подпространстве $\mathfrak{H}_{m}$ следующим образом:

$$
\widehat{H}_{\mathfrak{e}} \psi_{m}(y, \theta)=\widehat{H}_{\mathfrak{e} m} \psi_{m}(y, \theta)=\frac{1}{\sqrt{y}} \sqrt{\frac{\sigma}{2 \pi}} e^{i \sigma m \theta} \hat{h}_{\mathfrak{e} m} f_{m}(y),
$$

где $\hat{h}_{\mathfrak{e} m}$ - с. с. оператор, действующий в гильбертовом пространстве $\mathfrak{h}_{m} \equiv L^{2}\left(\mathbb{R}_{+}\right)$ и ассоциированный со следующей радиальной дифференциальной операцией $\check{h}_{m}$ :

$$
\begin{aligned}
& \check{h}_{\mathrm{O} m}=-\partial_{u}^{2}+u^{-2}\left(m^{2}-\frac{1}{4}\right)+\lambda u^{2}, \\
& \check{h}_{\mathrm{C} m}=-\partial_{x}^{2}+(2 x)^{-2}\left(m^{2}-1\right)+g x^{-1} .
\end{aligned}
$$

Соответствующие радиальные уравнения Шредингера имеют вид

$$
\check{h}_{\mathrm{O} m} f_{\mathrm{O} m}(u)=E_{\mathrm{O}} f_{\mathrm{O} m}(u), \quad \check{h}_{\mathrm{C} m} f_{\mathrm{C} m}(x)=E_{\mathrm{C}} f_{\mathrm{C} m}(x) .
$$

И наоборот, если мы построим с. с. операторы $\hat{h}_{\mathfrak{e} m}$ для каждого $m$, то все операторы $\widehat{H}_{\mathfrak{e} m}$, определяемые соотношением (19), являются с.с., и семейство с. с. операторов $\widehat{H}_{\mathfrak{e} m}, m \in \mathbb{Z}$, определяет полный с. с. оператор $\widehat{H}_{\mathfrak{e}}$ с помощью формулы (18). Таким образом, достаточно описать все с. с. операторы $\hat{h}_{\mathfrak{e} m}$ в подпространствах $\mathfrak{h}_{m}$, чтобы найти все с. с. операторы $\widehat{H}_{\mathfrak{e}}$, коммутирующие с $\widehat{S}_{\alpha}$, а спектральный анализ с. с. оператора $\widehat{H}_{\mathfrak{e} m}$ в пространстве $\mathfrak{H}_{m}$ сводится к спектральному анализу соответствующего с. с. оператора $\hat{h}_{\mathfrak{e} m}$ в пространстве $\mathfrak{h}_{m}$.

Решение такой спектральной задачи было рассмотрено в работе [7] (см. также работы [9], [10]). Мы приведем полученные в этих работах результаты в форме, которая позволит сравнить спектры и собственные функции дуальных теорий. В п. 3.2 рассмотрено уравнение Шредингера для осциллятора, в п. 3.3 - уравнение Шредингера для кулоноподобной системы. Результаты сравниваются в п. 3.4, где установлено взаимно однозначное соответствие спектров и (обобщенных) собственных функций с. с. гамильтонианов. 
3.1.3. С. с. гамильтонианы $\hat{h}_{\mathfrak{e} m}$. Для рассматриваемой дифференциальной операции область $D_{h_{\mathfrak{e} m}}$ с. с. операторов $\hat{h}_{\mathfrak{e} m}$ является подпространством так называемой натуральной области

$$
D_{\breve{h}_{m}}^{*}\left(\mathbb{R}_{+}\right)=\left\{\psi_{*}: \psi_{*}, \psi_{*}^{\prime} \text { а. н. в } \mathbb{R}_{+}, \quad \psi_{*}, \check{h}_{m} \psi_{*} \in L^{2}\left(\mathbb{R}_{+}\right)\right\} \text {. }
$$

Можно показать, что функции $\psi_{*} \in D_{\breve{h}_{m}}^{*}\left(\mathbb{R}_{+}\right)$имеют следующее асимптотическое поведение:

для обеих систем, осцилляторной и кулоноподобной,

$$
\begin{gathered}
\overline{\psi_{*}(y)} \psi_{*}^{\prime}(y)-\overline{\psi_{*}^{\prime}(y)} \psi_{*}(y) \rightarrow 0 \text { при } y \rightarrow \infty, \\
\psi_{*}(y), \psi_{*}^{\prime}(y) \rightarrow 0 \text { при } y \rightarrow 0 \text { для } \begin{cases}|m| \geqslant 1, & \check{h}_{m}=\check{h}_{\mathrm{O} m}, \\
|m| \geqslant 2, & \check{h}_{m}=\check{h}_{\mathrm{C} m} ;\end{cases}
\end{gathered}
$$

в случае осцилляторной системы при $u \rightarrow 0, m=0$

$$
\begin{aligned}
& \psi_{\mathrm{O} *}(u)=a_{1} o_{10, \text { as }}(u)+a_{2} o_{20, \text { as }}(u)+O\left(u^{3 / 2} \ln u\right), \\
& \psi_{\mathrm{O} *}^{\prime}(u)=a_{1} o_{10, \text { as }}^{\prime}(u)+a_{2} o_{20, \text { as }}^{\prime}(u)+O\left(u^{1 / 2} \ln u\right),
\end{aligned}
$$

где $o_{10, \text { as }}(u)=\sqrt{\kappa_{0} u}, o_{20, \text { as }}(u)=\sqrt{\kappa_{0} u} \ln \left(\kappa_{0} u\right)$;

в случае кулоноподобной системы при $x \rightarrow 0,|m|=1$

$$
\psi_{\mathrm{C} *}(x)=a_{1} \kappa_{0} x+a_{2} c_{21, \text { as }}(x)+O\left(x^{3 / 2}\right), \quad \psi_{\mathrm{C} *}^{\prime}(x)=a_{1} \kappa_{0}+a_{2} c_{21, \text { as }}^{\prime}(x)+O\left(x^{1 / 2}\right),
$$

где $c_{21, \text { as }}(x)=1+(\mathbf{C}-1) g x+g x \ln \left(\kappa_{0} x\right), \mathbf{C}-$ постоянная Эйлера,

при $x \rightarrow 0, m=0$

$$
\begin{aligned}
& \psi_{\mathrm{C} *}(x)=a_{1} c_{10, \text { as }}(x)+a_{2} c_{20, \text { as }}(x)+O\left(x^{3 / 2} \ln x\right), \\
& \psi_{\mathrm{C} *}^{\prime}(x)=a_{1} c_{10, \text { as }}^{\prime}(x)+a_{2} c_{20, \text { as }}^{\prime}(x)+O\left(x^{1 / 2} \ln x\right),
\end{aligned}
$$

где $c_{10, \text { as }}(x)=\left(\kappa_{0} x\right)^{1 / 2}, c_{20 \text {,as }}(x)=(1 / 2)\left(\kappa_{0} x\right)^{1 / 2} \ln \left(\kappa_{0} x\right)$.

Область $D_{h_{\mathfrak{e}}}$ содержит функции $\psi \in D_{\breve{h}_{m}}^{*}\left(\mathbb{R}_{+}\right)$, которые удовлетворяют дополнительным условиям

$$
\int_{0}^{\infty}\left[\overline{\psi(y)} \check{h}_{m} \psi(y)-\overline{\check{h}_{m} \psi(y)} \psi(y)\right] d y=\left.\left[\overline{\psi_{*}(y)} \psi_{*}^{\prime}(y)-\overline{\psi_{*}^{\prime}(y)} \psi_{*}(y)\right]\right|_{y \rightarrow 0}=0 .
$$

Отсюда вытекает, что существуют следующие семейства с. с. операторов $\hat{h}_{\mathfrak{e} m}$.

При $|m| \geqslant 1$ для $\check{h}_{m}=\check{h}_{\mathrm{O} m}$ и при $|m| \geqslant 2$ для $\check{h}_{m}=\check{h}_{\mathrm{C} m}$ для любого фиксированного $m$ существует единственный с. с. оператор $\hat{h}_{\mathfrak{e} m}$, область которого есть $D_{h_{\mathfrak{e} m}}=D_{\breve{h}_{m}}^{*}\left(\mathbb{R}_{+}\right)$.

При $m=0$ имеем семейство с. с. операторов $\hat{h}_{0 \zeta_{0}}$, зависящих от параметра $\zeta_{0}$, где $\left|\zeta_{0}\right| \leqslant \pi / 2, \zeta_{0}=-\pi / 2 \sim \zeta_{0}=\pi / 2$ : в этом случае область $D_{h_{0 \zeta_{0}}}$ состоит из функций $\psi \in D_{\breve{h}_{0}}^{*}\left(\mathbb{R}_{+}\right)$, удовлетворяющих следующим условиям: для осцилляторной системы при $u \stackrel{\rightarrow}{\longrightarrow}$

$$
\begin{aligned}
& \psi_{\mathrm{O} *}(u)=a\left[o_{10, \text { as }}(u) \sin \zeta_{0}+o_{20, \text { as }}(u) \cos \zeta_{0}\right]+O\left(u^{3 / 2} \ln u\right), \\
& \psi_{\mathrm{O} *}^{\prime}(u)=a\left[o_{10, \text { as }}^{\prime}(u) \sin \zeta_{0}+o_{20, \text { as }}^{\prime}(u) \cos \zeta_{0}\right]+O\left(u^{1 / 2} \ln u\right)
\end{aligned}
$$


для кулоноподобной системы при $x \rightarrow 0$

$$
\begin{aligned}
& \psi_{\mathrm{C} *}(x)=a\left[\kappa_{0} x \sin \zeta_{0}+c_{20, \text { as }}(x) \cos \zeta_{0}\right]+O\left(x^{3 / 2}\right), \\
& \psi_{\mathrm{C} *}^{\prime}(x)=a\left[\kappa_{0} \sin \zeta_{0}+c_{20, \text { as }}^{\prime}(x) \cos \zeta_{0}\right]+O\left(x^{1 / 2}\right) .
\end{aligned}
$$

Пусть $|m|=1, \check{h}_{ \pm 1}=\check{h}_{\mathrm{C}, \pm 1}$. Для $m=1$ имеем семейство с. с. операторов $\hat{h}_{\mathrm{C} \zeta_{1}}$, зависящих от параметра $\zeta_{1}$, где $\left|\zeta_{1}\right| \leqslant \pi / 2, \zeta_{1}=-\pi / 2 \sim \zeta_{1}=\pi / 2$. Для этих операторов область $D_{\check{h}_{\mathrm{C} \zeta_{1}}}$ состоит из функций $\psi \in D_{\breve{h}_{\mathrm{C} \zeta_{1}}}^{*}\left(\mathbb{R}_{+}\right)$, удовлетворяющих следующим условиям при $x \rightarrow 0$ :

$$
\begin{aligned}
& \psi_{\mathrm{C} *}(x)=a\left[\kappa_{0} x \sin \zeta_{1}+c_{21, \text { as }}(x) \cos \zeta_{1}\right]+O\left(x^{3 / 2}\right), \\
& \psi_{\mathrm{C} *}^{\prime}(u)=a\left[\kappa_{0} \sin \zeta_{1}+c_{21, \text { as }}^{\prime}(x) \cos \zeta_{1}\right]+O\left(x^{1 / 2}\right) .
\end{aligned}
$$

При $m=-1$ получаем те же результаты с формальной заменой $\zeta_{1}$ на $\zeta_{-1}$.

В то время как осцилляторная система при $|m|=1$ имеет только один с. с. гамильтониан, кулоноподобная система имеет целое семейство с. с. операторов. С технической точки зрения эту ситуацию можно объяснить следующим образом. Безусловно, в общем пространстве функций осцилляторной системы есть функции с асимптотическим поведением при $u \rightarrow 0$, дуальным (см. соотношения (17)) к поведению, заданному в (25):

$$
\psi_{\mathrm{O}}(u)=a\left[\left(\kappa_{0} u\right)^{3 / 2} \sin \zeta_{1}+\left(\kappa_{0} u\right)^{-1 / 2} \cos \zeta_{1}\right]+O\left(u^{3 / 2}\right)
$$

Однако функции с таким асимптотическим поведением могут принадлежать некоторой натуральной области (или даже пространству $\left.L^{2}\left(\mathbb{R}_{+}\right)\right)$только при $\zeta_{1}= \pm \pi / 2$, так как в противном случае функции с асимптотическим поведением (26) не являются квадратично-интегрируемыми в начале координат. Таким образом получаем, что для $m=1$ только одна кулоноподобная система может быть дуальна осцилляторной системе, она описывается гамильтонианом с областью, заданной условиями (25) при $\zeta_{1}= \pm \pi / 2$. Аналогично при $m=-1$ только одна кулоноподобная система при $\zeta_{1}= \pm \pi / 2$ может быть дуальна осцилляторной системе.

Можно показать, что спектр любого с. с. гамильтониана $\hat{h}_{\mathfrak{e} m}$ простой (невырожденный).

3.2. Квантовый двумерный осциллятор. Напомним, что радиальный гамильтониан действует как дифференциальная операция (20).

Удобно положить

$$
\varkappa=\left\{\begin{array}{ll}
\lambda^{1 / 4}, & \lambda \geqslant 0, \\
e^{-i \pi / 4}|\lambda|^{1 / 4}, & \lambda<0,
\end{array} \quad \varkappa^{2}= \begin{cases}\lambda^{1 / 2}, & \lambda \geqslant 0, \\
e^{-i \pi / 2}|\lambda|^{1 / 2}, & \lambda<0 .\end{cases}\right.
$$

Введем также обозначения

$$
\begin{gathered}
\rho=\rho\left(u ; \lambda, E_{0}\right)=(\varkappa u)^{2}, \\
w_{\mathrm{O}}=w_{\mathrm{O}}\left(\lambda, E_{\mathrm{O}}\right)=\frac{E_{\mathrm{O}}}{4 \sqrt{\lambda}}, \\
\alpha_{\mathrm{O} m}=\alpha_{\mathrm{O} m}\left(\lambda, E_{\mathrm{O}}\right)=\frac{1}{2}+\frac{|m|}{2}-w_{\mathrm{O}}\left(\lambda, E_{\mathrm{O}}\right), \quad \beta_{m}=1+|m|, \\
\Omega_{\mathrm{O}}\left(\lambda, E_{\mathrm{O}}\right)=\ln \frac{\kappa_{0}^{2}}{\sqrt{\lambda}}+2 \psi(1)-\psi\left(\alpha_{\mathrm{O}, 0}\left(\lambda, E_{\mathrm{O}}\right)\right)
\end{gathered}
$$


и функции

$$
\begin{aligned}
O_{m}\left(u ; \lambda, E_{\mathrm{O}}\right) & =\left(\kappa_{0} u\right)^{1 / 2+|m|} e^{-\rho / 2} \Phi\left(\alpha_{\mathrm{O} m}\left(\lambda, E_{\mathrm{O}}\right), \beta_{m} ; \rho\right), \quad|m| \geqslant 1, \\
O_{\zeta}\left(u ; \lambda, E_{\mathrm{O}}\right) & =O_{10}\left(u ; \lambda, E_{\mathrm{O}}\right) \sin \zeta+O_{20}\left(u ; \lambda, E_{\mathrm{O}}\right) \cos \zeta, \\
O_{10}\left(u ; \lambda, E_{\mathrm{O}}\right) & =\left(\kappa_{0} u\right)^{1 / 2} e^{-\rho / 2} \Phi\left(\alpha_{\mathrm{O}, 0}, 1 ; \rho\right), \\
O_{20}\left(u ; \lambda, E_{\mathrm{O}}\right) & =\left.\left(\kappa_{0} u\right)^{1 / 2} e^{-\rho / 2} \partial_{\delta} \Phi\left(\alpha_{\mathrm{O} \delta}, \beta_{\delta} ; \rho\right)\right|_{\delta=+0}+O_{10}\left(u ; \lambda, E_{\mathrm{O}}\right) \ln \left(\kappa_{0} u\right),
\end{aligned}
$$

где $\alpha_{\mathrm{O} \delta}=\alpha_{\mathrm{O}, 0}+\delta / 2$ и $\beta_{\delta}=1+\delta$. При этом в пределе $u \rightarrow 0$

$$
\begin{aligned}
O_{10}\left(u ; \lambda, E_{\mathrm{O}}\right) & =o_{10, \text { as }}(u)\left(1+O\left(u^{2}\right)\right), \\
O_{20}\left(u ; \lambda, E_{\mathrm{O}}\right) & =o_{20, \text { as }}(u)\left(1+O\left(u^{2}\right)\right), \\
O_{\zeta}\left(u ; \lambda, E_{\mathrm{O}}\right) & =o_{10, \text { as }}(u) \sin \zeta+o_{20, \text { as }}(u) \cos \zeta+O\left(u^{5 / 2} \ln u\right) .
\end{aligned}
$$

Функции $O_{m}, O_{10}$ и $O_{20}$ являются решениями уравнений $(22)$ (с соответствующими значениями $m), O_{\zeta}\left(u ; \lambda, E_{\mathrm{O}}\right)$ является решением уравнения $(22)$ при $m=0$, удовлетворяющим граничным условиям (23).

3.2.1. Спектр: случай $|m| \geqslant 1, \lambda>0$. В данном случае спектр с. с. гамильтониана $\hat{h}_{\mathfrak{e} m}$ является чисто дискретным,

$$
\operatorname{spec} \hat{h}_{\mathrm{Oem}}=\left\{E_{\mathrm{O} m, n}(\lambda)=2 \sqrt{\lambda}(1+|m|+2 n), n \in \mathbb{Z}_{+}\right\},
$$

а полная система (обобщенных) ортонормированных собственных функций есть

$$
\left\{U_{m, n}(u ; \lambda)=Q_{\mathrm{O} m, n}(\lambda) O_{m}\left(u ; \lambda, E_{\mathrm{O} m, n}(\lambda)\right), n \in \mathbb{Z}_{+}\right\},
$$

где

$$
Q_{\mathrm{O} m, n}(\lambda)=\frac{\left(\lambda^{1 / 4} / \kappa_{0}\right)^{|m|}}{|m| !} \sqrt{\frac{2 \sqrt{\lambda}(|m|+n) !}{\kappa_{0} n !}} .
$$

Заметим, что дискретные собственные значения $E_{\mathrm{O} m, n}(\lambda)$ являются корнями уравнений

$$
\begin{gathered}
f_{2, \mathrm{O} m, n}\left(\lambda, E_{\mathrm{O}}\right)=0, \\
f_{2, \mathrm{O} m, n}\left(\lambda, E_{\mathrm{O}}\right)=\alpha_{\mathrm{O} m}\left(\lambda, E_{\mathrm{O}}\right)+n, \quad|m| \geqslant 1, \quad n \in \mathbb{Z}_{+}
\end{gathered}
$$

(см. определения (27)).

3.2.2. Спектр: случай $|m| \geqslant 1, \lambda<0$. В данном случае спектр с.с. гамильтониана $\hat{h}_{\mathrm{Oem}}$ является чисто непрерывным и заполняет всю действительную ось, $\operatorname{spec} \hat{h}_{\mathrm{Oem}}=\mathbb{R}$, а полная система (обобщенных) ортонормированных собственных функций есть

$$
\left\{U_{m, E_{\mathrm{O}}}(u ; \lambda)=\rho_{\mathrm{O} m}\left(\lambda, E_{\mathrm{O}}\right) O_{m}\left(u ; \lambda, E_{\mathrm{O}}\right)\right\}
$$

где

$$
\begin{gathered}
O_{m}\left(u ; \lambda, E_{\mathrm{O}}\right)=\left(\kappa_{0} u\right)^{1 / 2+|m|} e^{-\rho / 2} \Phi\left(\alpha_{\mathrm{O} m}, \beta_{m} ; \rho\right), \quad \rho=-i\left(|\lambda|^{1 / 4} u\right)^{2}, \\
\rho_{\mathrm{O} m}\left(\lambda, E_{\mathrm{O}}\right)=\frac{\left(|\lambda|^{1 / 4} / \kappa_{0}\right)^{|m|}\left|\Gamma\left(\alpha_{\mathrm{O}}\right)\right| e^{\pi \tilde{w}_{\mathrm{O}} / 2}}{2 \sqrt{\pi \kappa_{0}}|m| !}
\end{gathered}
$$


и в данном случае величины (27) записываются как

$$
\alpha_{\mathrm{O} m}=\alpha_{\mathrm{O} m}\left(\lambda, E_{\mathrm{O}}\right)=\frac{1}{2}+\frac{|m|}{2}-i \widetilde{w}_{\mathrm{O}}, \quad \widetilde{w}_{\mathrm{O}}=\widetilde{w}_{\mathrm{O}}\left(\lambda, E_{\mathrm{O}}\right)=\frac{E}{4 \sqrt{|\lambda|}} .
$$

3.2.3. Спектр: случай $|m| \geqslant 1, \lambda=0$. Заметим, что в этом случае решения соответствующего уравнения (22) выражаются в терминах функций Бесселя. Для спектра и полной системы собственных функций получаем, что спектр с. с. гамильтониана $\hat{h}_{\mathrm{Oem}}$ чисто непрерывен и заполняет неотрицательную действительную полуось,

$$
\operatorname{spec} \hat{h}_{\mathrm{Oem}}=\mathbb{R}_{+},
$$

а полный набор (обобщенных) ортонормированных собственных функций есть

$$
\left\{U_{m, E_{\mathrm{O}}}(u)=\rho_{\mathrm{O} m}\left(E_{\mathrm{O}}\right) O_{m}\left(u ; E_{\mathrm{O}}\right)=\sqrt{\frac{u}{2}} J_{|m|}\left(E^{1 / 2} u\right), E_{\mathrm{O}} \in \mathbb{R}_{+}\right\},
$$

где

$$
\begin{gathered}
O_{m}\left(u ; E_{\mathrm{O}}\right)= \\
\left.O_{m}\left(u ; \lambda, E_{\mathrm{O}}\right)\right|_{\lambda \rightarrow 0}=\left(\frac{E^{1 / 2}}{2 \kappa_{0}}\right)^{-|m|}|m| !\left(\kappa_{0} u\right)^{1 / 2} J_{|m|}\left(E^{1 / 2} u\right) \\
\rho_{\mathrm{O} m}\left(E_{\mathrm{O}}\right)=\left.\rho_{\mathrm{O} m}\left(E_{\mathrm{O}}, \lambda\right)\right|_{\lambda \rightarrow-0}=\frac{\left(\sqrt{E} / 2 \kappa_{0}\right)^{|m|}}{\sqrt{2 \kappa_{0}}|m| !}
\end{gathered}
$$

Заметим также, что

$$
\begin{array}{rlll}
\rho_{\mathrm{O} m}\left(E_{\mathrm{O}}, \lambda\right)=0 & \text { при } & \lambda \rightarrow-0, \quad E_{\mathrm{O}}<0, \\
\rho_{\mathrm{O} m}\left(E_{0}, \lambda\right)=0 & \text { при } & \lambda<0 .
\end{array}
$$

3.2.4. Спектр: случай $m=0, \lambda>0$. При любом фиксированном $\zeta$ спектр оператора $\hat{h}_{\mathrm{O} \zeta}$ дискретный,

$$
\operatorname{spec} \hat{h}_{\mathrm{O} 0 \zeta}=\left\{E_{\mathrm{O} 0, n}(\lambda, \zeta), n \in \mathbb{Z}_{+}\right\} .
$$

Для $\zeta= \pm \pi / 2$ имеем $\operatorname{spec} \hat{h}_{\mathrm{O} 0, \pm \pi / 2}=\left\{\mathcal{E}_{\mathrm{O}, n}(\lambda), n \in \mathbb{Z}_{+}\right\}$, где

$$
\mathcal{E}_{\mathrm{O}, n}(\lambda)=2 \sqrt{\lambda}(1+2 n) .
$$

При $|\zeta|<\pi / 2$ значения $E_{\mathrm{O}, n}(\lambda, \zeta)$ являются решениями уравнения

$$
f_{2, \mathrm{O} \zeta}\left(\lambda, E_{\mathrm{O}}\right)=0, \quad f_{2, \mathrm{O} \zeta}\left(\lambda, E_{\mathrm{O}}\right)=\frac{1}{2} \Omega_{\mathrm{O}}\left(\lambda, E_{\mathrm{O}}\right)+\operatorname{tg} \zeta
$$

(см. определения $(27))$. Функция $f_{2, \mathrm{O}}(\lambda, E)$ обладает следующими свойствами:

$$
\begin{aligned}
& f_{2, \mathrm{O} \zeta}(\lambda, E) \rightarrow-\infty \quad \text { при } \quad E \rightarrow \mathcal{E}_{\mathrm{O},-1} \equiv-\infty, \quad \partial_{E} f_{2, \mathrm{O} \zeta}(\lambda, E)>0, \\
& \left.f_{2, \mathrm{O} \zeta}(\lambda, E)\right|_{E=\mathcal{E}_{\mathrm{O}, n}(\lambda) \pm 0}=\mp \infty, \quad n \in \mathbb{Z}_{+},
\end{aligned}
$$

при этом

$$
\partial_{\zeta} E_{\mathrm{O} 0, n}(\lambda, \zeta)<0, \quad \partial_{\lambda} E_{\mathrm{O} 0, n}(\lambda, \zeta)>0
$$

При фиксированном $\lambda$ в интервале значений $\left(\mathcal{E}_{\mathrm{O}, n-1}(\lambda), \mathcal{E}_{\mathrm{O}, n}(\lambda)\right), n \in \mathbb{Z}_{+}$, и при фиксированном $\zeta \in(-\pi / 2, \pi / 2)$ существует один уровень $E_{\mathrm{O}, n}(\lambda, \zeta)$, монотонно 
возрастающий от значения $\mathcal{E}_{\mathrm{O}, n-1}(\lambda)+0$ до $\mathcal{E}_{\mathrm{O}, n}(\lambda)-0$, когда $\zeta$ убывает от $\pi / 2-0$ до $-\pi / 2+0$. Заметим, что справедливы равенства

$$
\lim _{\zeta \rightarrow-\pi / 2} E_{\mathrm{O} 0, n}(\lambda, \zeta)=\lim _{\zeta \rightarrow \pi / 2} E_{\mathrm{O} 0, n+1}(\lambda, \zeta)=\mathcal{E}_{\mathrm{O}, n}(\lambda)
$$

которые говорят об эквивалентности расширений при $\zeta=-\pi / 2$ и $\zeta=\pi / 2$.

При фиксированном $\zeta$ функция $E_{\mathrm{O}, n}(\lambda, \zeta)$ параметра $\lambda$ ведет себя следующим образом. Для $n \geqslant 1$ при любом $\zeta$ она монотонно возрастает от 0 до $\infty$, когда $\lambda$ меняется от 0 до $\infty$. При $n=0$ функция $E_{\mathrm{O}, 0}(\lambda, \zeta)$, когда $\lambda$ растет от 0 до $\infty$, монотонно возрастает от значения

$$
E_{\mathrm{O} 0,0}(0, \zeta)=-4 \kappa_{0}^{2} e^{2(\operatorname{tg} \zeta-\mathbf{C})}
$$

до $\infty$, пересекая ось $\lambda$ (т. е. $\left.E_{\mathrm{O}}=0\right)$ в точке

$$
\lambda_{\zeta}=\kappa_{0}^{4} e^{4(\operatorname{tg} \zeta-\mathbf{C})-2 \psi(1 / 2)}
$$

Полный набор ортонормированных собственных функций (для любых $\zeta$ ) задается формулами

$$
\begin{aligned}
U_{0 \zeta, n}(u ; \lambda) & =Q_{\mathrm{O} \zeta 0, n}(\lambda) O_{\zeta}\left(u ; \lambda, E_{\mathrm{O} 0, n}(\lambda, \zeta)\right), \\
Q_{\mathrm{O} \zeta 0, n}(\lambda) & = \begin{cases}\sqrt{\frac{2 \lambda^{1 / 2}}{\kappa_{0}}}, & \zeta \in \mathbb{Z}_{+}, \\
{\left[\kappa_{0}^{3 / 2} \partial_{E} f_{2 \mathrm{O} \zeta}\left(\lambda, E_{\mathrm{O} 0, n}(\lambda, \zeta)\right) \cos ^{2} \zeta\right]^{-1 / 2},} & |\zeta|<\frac{\pi}{2}\end{cases}
\end{aligned}
$$

3.2.5. Спектр: случай $m=0, \lambda<0$. В данном случае спектр гамильтониана $\hat{h}_{\text {Оо }}$ является непрерывным и заполняет всю действительную ось, spec $\hat{h}_{\mathrm{O} \zeta}=\mathbb{R}$. Полная система (обобщенных) ортонормированных собственных функций состоит из функций

$$
U_{0 \zeta, E_{\mathrm{O}}}(u ; \lambda)=\rho_{\mathrm{O} 0 \zeta}\left(\lambda, E_{\mathrm{O}}\right) O_{\zeta}\left(u ; \lambda, E_{\mathrm{O}}\right), \quad E_{\mathrm{O}} \in \mathbb{R}
$$

где

$$
\begin{aligned}
\rho_{\mathrm{O} 0 \zeta}^{2}\left(\lambda, E_{\mathrm{O}}\right)=\frac{4}{\kappa_{0}} & \frac{B_{\mathrm{O}}\left(\lambda, E_{\mathrm{O}}\right)}{4\left(A_{\mathrm{O}}\left(\lambda, E_{\mathrm{O}}\right) \cos \vartheta+2 \sin \vartheta\right)^{2}+\pi^{2} B_{\mathrm{O}}^{2}\left(\lambda, E_{\mathrm{O}}\right) \cos ^{2} \vartheta}, \\
A_{\mathrm{O}}\left(\lambda, E_{\mathrm{O}}\right) & =\operatorname{Re} \Omega_{\mathrm{O}}\left(\lambda, E_{\mathrm{O}}\right), \\
B_{\mathrm{O}}\left(\lambda, E_{\mathrm{O}}\right) & =\frac{2}{\pi} \operatorname{Im} \Omega_{\mathrm{O}}\left(\lambda, E_{\mathrm{O}}\right)=1+\operatorname{th}\left(\pi \widetilde{w}_{\mathrm{O}}\left(\lambda, E_{\mathrm{O}}\right)\right)>0
\end{aligned}
$$

3.2.6. Спектр: случай $m=0, \lambda=0$. Заметим, что в данном случае решения уравнений (22) выражаются в терминах функций Бесселя.

При $\zeta= \pm \pi / 2$ имеем spec $\hat{h}_{\mathrm{O} 0, \pm \pi / 2}=\mathbb{R}_{+}$, множество

$$
\left\{U_{0, \pm \pi / 2, E_{\mathrm{O}}}(u)=\rho_{\mathrm{O} 0, \pm \pi / 2}\left(0, E_{\mathrm{O}}\right) O_{10}(u ; 0, E)=\sqrt{\frac{u}{2}} J_{0}(p u), E_{\mathrm{O}} \in \mathbb{R}_{+}\right\}
$$


(обобщенных) собственных функций оператора $\hat{h}_{\mathrm{O}, \pm \pi / 2}$ образует полную ортонормированную систему в пространстве $L^{2}\left(\mathbb{R}_{+}\right)$: здесь

$$
\begin{gathered}
O_{10}\left(u ; 0, E_{\mathrm{O}}\right)=\lim _{\lambda \rightarrow 0} O_{10}\left(u ; \lambda, E_{\mathrm{O}}\right)=\sqrt{\kappa_{0} u} J_{0}\left(p_{\mathrm{O}} u\right), \quad p_{\mathrm{O}}=\sqrt{E_{\mathrm{O}}} \\
\rho_{\mathrm{O} 0, \pm \pi / 2}\left(0, E_{\mathrm{O}}\right)=\frac{1}{\sqrt{2 \kappa_{0}}} .
\end{gathered}
$$

При $|\zeta|<\pi / 2$ спектр оператора $\hat{h}_{\text {Оо }}$ состоит из непрерывной части и дискретного уровня, энергию которого мы обозначим через $E_{\mathrm{O}, 0}(0, \zeta)$ :

$$
\operatorname{spec} \hat{h}_{\mathrm{O} 0, \pm \pi / 2}=\mathbb{R}_{+} \cup\left\{E_{\mathrm{O} 0,0}(0, \zeta)\right\},
$$

где

$$
E_{\mathrm{O} 0,0}(0, \zeta)=-\frac{4}{\kappa_{0}^{2}} e^{2(\operatorname{tg} \zeta-\mathbf{C})}
$$

Множество

$$
\left\{U_{0 \zeta, E_{\mathrm{O}}}(u)=\rho_{\mathrm{O} 0 \zeta}\left(0, E_{\mathrm{O}}\right) O_{\zeta}\left(u ; 0, E_{\mathrm{O}}\right), E_{\mathrm{O}} \in \mathbb{R}_{+}, U_{0 \zeta}(u)=Q_{\mathrm{O} 0 \zeta} O_{\zeta}\left(u ; 0, E_{\mathrm{O} 0,0}(0, \zeta)\right)\right\}
$$

(обобщенных)собственных функций оператора $\hat{h}_{\mathrm{O}}$ об образует полную ортонормированную систему в $L^{2}\left(\mathbb{R}_{+}\right)$. Здесь

$$
\begin{gathered}
\rho_{\mathrm{O} 0 \zeta}^{2}\left(0, E_{\mathrm{O}}\right)=\lim _{\lambda \rightarrow 0} \rho_{\mathrm{O} 0 \zeta}^{2}\left(\lambda, E_{\mathrm{O}}\right)=\frac{2}{\kappa_{0}} \frac{1}{\left[g\left(E_{\mathrm{O}}\right) \cos \zeta+2 \sin \zeta\right]^{2}+\pi^{2} \cos ^{2} \zeta}, \\
g\left(E_{\mathrm{O}}\right)=2 \psi(1)-\ln \frac{E_{\mathrm{O}}}{4 \kappa_{0}^{2}}, \\
Q_{\mathrm{O} 0 \zeta}=\sqrt{\frac{2\left|E_{\mathrm{O} 0,0}(0, \zeta)\right|}{\kappa_{0} \cos ^{2} \zeta}, \quad O_{\zeta}\left(u ; 0, E_{\mathrm{O}}\right)=O_{10}\left(u ; 0, E_{\mathrm{O}}\right) \sin \zeta+O_{20}\left(u ; 0, E_{\mathrm{O}}\right) \cos \zeta,} \\
O_{20}\left(u ; 0, E_{\mathrm{O}}\right)=\lim _{\lambda \rightarrow 0} O_{20}\left(u ; \lambda, E_{\mathrm{O}}\right)= \\
=\left(\kappa_{0} u\right)^{1 / 2} J_{0}(k u) \ln \left(\kappa_{0} u\right)+\left(\kappa_{0} u\right)^{1 / 2} Q\left(E_{\mathrm{O}} u^{2}\right), \quad k= \begin{cases}\sqrt{E_{\mathrm{O}},} & E_{\mathrm{O}} \geqslant 0, \\
i \sqrt{\left|E_{\mathrm{O}}\right|}, & E_{\mathrm{O}}<0,\end{cases}
\end{gathered}
$$

где

$$
Q(z)=\sum_{j=1}^{\infty} \frac{(-1)^{j}}{(j !)^{2}}\left(\frac{z}{4}\right)^{j}[\psi(1)-\psi(j+1)], \quad Q(z)=O(z), \quad z \rightarrow 0 .
$$

Заметим, что результаты данного пункта можно получить в пределе $\lambda \rightarrow 0$ из результатов предыдущего пункта, а также путем непосредственного решения уравнения Шредингера при $\lambda=0$. Более того, в пределе $\lambda \rightarrow 0$ дискретный уровень для $\zeta= \pm \pi / 2$ и $\lambda>0$ "покидает" спектр: область локализации этих волновых функций уходит на бесконечность, и они становятся ортогональными всем функциям из пространства $L^{2}\left(\mathbb{R}_{+}\right)$. Кроме того, значения дискретных уровней были обозначены как $E_{\mathrm{O}, 0}(0, \zeta)$ специально. Это те значения, к которым стремится собственное значение $E_{\mathrm{O}, 0}(\lambda, \zeta)$ дискретного уровня при $\lambda>0, n=0$, когда $\lambda \rightarrow 0$. 
3.3. Квантовое двумерное кулоноподобное взаимодействие. Напомним, что радиальный гамильтониан действует как дифференциальная операция (21).

Введем обозначения

$$
\begin{gathered}
p_{\mathrm{C}}=\sqrt{E_{\mathrm{C}}} \text { для } E_{\mathrm{C}} \geqslant 0, \quad \tau_{\mathrm{C}}=\sqrt{\left|E_{\mathrm{C}}\right|} \text { для } E_{\mathrm{C}}<0, \\
K=\left\{\begin{array}{ll}
-i p_{\mathrm{C}}, & E_{\mathrm{C}} \geqslant 0, \\
\tau_{\mathrm{C}}, & E_{\mathrm{C}}<0,
\end{array} \quad z=z\left(x ; g, E_{\mathrm{C}}\right)=2 K x\right. \\
w_{\mathrm{C}}\left(g, E_{\mathrm{C}}\right)=-\frac{g}{2 K}=\left\{\begin{array}{ll}
i \widetilde{w}_{\mathrm{C}}, & E_{\mathrm{C}} \geqslant 0, \\
-\frac{g}{2 \tau_{\mathrm{C}}}, & E_{\mathrm{C}}<0,
\end{array} \widetilde{w}_{\mathrm{C}}\left(g, E_{\mathrm{C}}\right)=-\frac{g}{2 p_{\mathrm{C}}},\right. \\
\alpha_{\mathrm{C} m}=\alpha_{\mathrm{C} m}\left(g, E_{\mathrm{C}}\right)=\frac{1}{2}+\frac{|m|}{2}-w_{\mathrm{C}}\left(g, E_{\mathrm{C}}\right), \quad \beta_{m}=1+|m|, \\
\Omega_{\mathrm{C}}\left(g, E_{\mathrm{C}}\right)=\ln \frac{\kappa_{0}}{2 K}+2 \psi(1)-\psi\left(\alpha_{\mathrm{C}, 0}\left(g, E_{\mathrm{C}}\right)\right)
\end{gathered}
$$

и функции

$$
\begin{aligned}
C_{m}\left(x ; g, E_{\mathrm{C}}\right) & =\left(\kappa_{0} x\right)^{1 / 2+|m| / 2} e^{-z / 2} \Phi\left(\alpha_{\mathrm{C} m}, \beta_{m} ; z\right), \quad m \in \mathbb{Z}, \\
C_{\zeta}\left(x ; g, E_{\mathrm{C}}\right) & =C_{10}\left(x ; g, E_{\mathrm{C}}\right) \sin \zeta+C_{20}\left(x ; g, E_{\mathrm{C}}\right) \cos \zeta, \\
C_{10}\left(x ; g, E_{\mathrm{C}}\right) & =\left(\kappa_{0} x\right)^{1 / 2} e^{-z / 2} \Phi\left(\alpha_{\mathrm{C}, 0}, 1 ; z\right), \\
C_{20}\left(x ; g, E_{\mathrm{C}}\right) & =\left.\left(\kappa_{0} x\right)^{1 / 2} e^{-z / 2} \partial_{\delta} \Phi\left(\alpha_{C \delta}, \beta_{\delta} ; z\right)\right|_{\delta=+0}+\frac{1}{2} C_{10}\left(x ; E_{\mathrm{C}}\right) \ln \left(\kappa_{0} x\right),
\end{aligned}
$$

где $\alpha_{C \delta}=\alpha_{\mathrm{C} 0}+\delta / 2$ и $\beta_{\delta}=1+\delta$. При этом в пределе $x \rightarrow 0$

$$
\begin{aligned}
C_{10}\left(x ; g, E_{\mathrm{C}}\right) & =c_{10, \text { as }}(x)(1+O(x)), \\
C_{20}\left(x ; g, E_{\mathrm{C}}\right) & =c_{20, \text { as }}(x)(1+O(x \ln x)), \\
C_{\zeta}\left(x ; g, E_{\mathrm{C}}\right) & =c_{10, \text { as }}(x) \sin \zeta+c_{20, \text { as }}(x) \cos \zeta+O\left(x^{3 / 2} \ln x\right),
\end{aligned}
$$

Функции $C_{m}, C_{10}$, и $C_{20}$ являются решениями уравнения (22) (с соответствующими значениями $m)$, функция $C_{\zeta}\left(u ; \lambda, E_{\mathrm{O}}\right)$ - решение уравнения $(22)$ (при $m=0$ ), удовлетворяющее граничному условию (24).

3.3.1. Спектр: случай $|m| \geqslant 1, g \geqslant 0$. Заметим, что при $|m|=1$ мы рассматриваем с. с. радиальный гамильтониан только при значении параметра $\zeta= \pm \pi / 2$.

Получаем, что в данном случае спектр с.с. гамильтониана $\hat{h}_{\mathrm{Cem}}$ чисто непрерывный и заполняет неотрицательную действительную полуось, spec $\hat{h}_{\mathrm{Ce} m}=\mathbb{R}_{+}$, a полная система (обобщенных) ортонормированных собственных функций задается каK

$$
\left\{X_{m, E_{\mathrm{C}}}(x ; g)=\rho_{\mathrm{C} m}\left(g, E_{\mathrm{C}}\right) C_{m}\left(x ; g, E_{\mathrm{C}}\right), E_{\mathrm{C}} \in \mathbb{R}_{+}\right\},
$$

где

$$
\rho_{\mathrm{C} m}\left(g, E_{\mathrm{C}}\right)=\frac{|\Gamma(\alpha)|\left(2 p_{\mathrm{C}}\right)^{|m| / 2} e^{-\pi g / 4 p_{\mathrm{C}}}}{\sqrt{2 \pi} \kappa_{0}^{1 / 2+|m| / 2}|m| !}
$$

и в данном случае величины (30) могут быть записаны как

$$
z=-2 i p x, \quad \alpha_{\mathrm{C} m}=\frac{1}{2}+\frac{|m|}{2}-i \widetilde{w}_{\mathrm{C}}
$$


3.3.2. Спектр: случай $|m| \geqslant 1, g<0$. В этом случае спектр с. с. гамильтониана $\hat{h}_{\mathrm{Cem}}$ содержит непрерывную часть и, вдобавок, дискретную часть,

$$
\operatorname{spec} \hat{h}_{\mathrm{Ce} m}=\mathbb{R}_{+} \cup\left\{E_{\mathrm{C} m, n}(g)<0, n \in \mathbb{Z}_{+}\right\},
$$

и полная система (обобщенных) ортонормированных собственных функций состоит из функций

$$
\begin{aligned}
X_{m, E_{\mathrm{C}}}(x ; g) & =\rho_{\mathrm{C} m}\left(g, E_{\mathrm{C}}\right) C_{m}\left(x ; g, E_{\mathrm{C}}\right), & E_{\mathrm{C}} & \in \mathbb{R}_{+}, \\
X_{m, n}(x ; g) & =Q_{\mathrm{C} m, n}(g) C_{m}\left(x ; g, E_{\mathrm{C} m, n}(g)\right), & n & \in \mathbb{Z}_{+},
\end{aligned}
$$

где в данном случае

$$
\begin{gathered}
z=\left\{\begin{array}{ll}
-2 i p x, & E_{\mathrm{C}} \geqslant 0, \\
2 \sqrt{\left|E_{\mathrm{C}}\right|} x, & E_{\mathrm{C}}<0,
\end{array} \quad \alpha_{\mathrm{C} m}\left(g, E_{\mathrm{C}}\right)=\left\{\begin{array}{l}
\frac{1}{2}+\frac{|m|}{2}-i \widetilde{w}_{\mathrm{C}}, \quad E_{\mathrm{C}} \geqslant 0, \\
\frac{1}{2}+\frac{|m|}{2}+\frac{g}{2 \tau}, \quad E_{\mathrm{C}}<0,
\end{array} \quad \tau=2 \sqrt{\left|E_{\mathrm{C}}\right|,}\right.\right. \\
E_{\mathrm{C} m, n}(g)=-\frac{g^{2}}{(1+|m|+2 n)^{2}}=-\tau_{m, n}^{2}, \\
Q_{\mathrm{C} m, n}(g)=\left(\frac{2 \tau_{m, n}}{\kappa_{0}}\right)^{1 / 2+|m| / 2} \sqrt{\frac{2 \tau_{m, n} \Gamma(1+n+|m|)}{(1+|m|+2 n) \Gamma(1+n)}} .
\end{gathered}
$$

Дискретные собственные значения $E_{\mathrm{C} m, n}(g)$ являются корнями уравнения

$$
\begin{gathered}
f_{2, \mathrm{C} m, n}\left(g, E_{\mathrm{C}}\right)=0, \\
f_{2, \mathrm{C} m, n}\left(g, E_{\mathrm{C}}\right)=\alpha_{\mathrm{C} m}\left(g, E_{\mathrm{C}}\right)+n, \quad m \geqslant 1, \quad n \in \mathbb{Z}_{+} .
\end{gathered}
$$

3.3.3. Спектр: случай $m=0, \zeta= \pm \pi / 2$. В данном случае при $g \geqslant 0$ спектр гамильтониана $\hat{h}_{\mathrm{C} 0, \pm \pi / 2}$ непрерывный, spec $\hat{h}_{\mathrm{C} 0, \pm \pi / 2}=\mathbb{R}_{+}$, и (обобщенные) собственные функции

$$
X_{0, \pm \pi / 2, E_{\mathrm{C}}}(x ; g)=\rho_{\mathrm{C} 0, \pm \pi / 2}\left(g, E_{\mathrm{C}}\right) C_{10}\left(x ; g, E_{\mathrm{C}}\right), \quad E_{\mathrm{C}} \in \mathbb{R}_{+}
$$

где

$$
\rho_{\mathrm{C} 0, \pm \pi / 2}\left(g, E_{\mathrm{C}}\right)=\frac{1}{\sqrt{2 \kappa_{0}}}\left[1-\operatorname{th} \frac{\pi g}{2 p}\right]^{1 / 2},
$$

образуют полную ортонормированную систему в пространстве $L^{2}\left(\mathbb{R}_{+}\right)$. При $g<0$ спектр оператора $\hat{h}_{\mathrm{C} 0, \pm \pi / 2}$ содержит непрерывную и дискретные части,

$$
\operatorname{spec} \hat{h}_{\mathrm{C} 0, \pm \pi / 2}=\mathbb{R}_{+} \cup\left\{E_{\mathrm{C}, n}(g)<0, n \in \mathbb{Z}_{+}\right\},
$$

и (обобщенные) собственные функции

$$
\begin{aligned}
X_{0, \pm \pi / 2, E_{\mathrm{C}}}(x ; g) & =\rho_{\mathrm{C} 0, \pm \pi / 2}\left(g, E_{\mathrm{C}}\right) C_{10}\left(x ; g, E_{\mathrm{C}}\right), & E_{\mathrm{C}} & \in \mathbb{R}_{+}, \\
X_{0, \pm \pi / 2, n}(x ; g) & =Q_{\mathrm{C} 0, \pm \pi / 2, n}(g) C_{10}\left(x ; g, E_{\mathrm{C}, n}(g)\right), & n & \in \mathbb{Z}_{+}
\end{aligned}
$$


образуют полную ортонормированную систему в пространстве $L^{2}\left(\mathbb{R}_{+}\right)$. Здесь

$$
E_{\mathrm{C}, n}(g)=-\frac{g^{2}}{(1+2 n)^{2}}, \quad Q_{\mathrm{C} 0, \pm \pi / 2, n}(g)=\frac{2|g|}{\sqrt{\kappa_{0}}(1+2 n)^{3 / 2}} .
$$

Заметим, что эти результаты для спектров и собственных функций можно получить из соответствующих результатов предыдущих пунктов формальной заменой $|m|=0$.

3.3.4. Спектр: случай $m=0,|\zeta|<\pi / 2$. При $g>0$ имеем

$$
\operatorname{spec} \hat{h}_{\mathrm{C} 0 \zeta}= \begin{cases}\mathbb{R}_{+}, & \zeta \in\left(-\pi / 2, \zeta_{g}\right), \\ \mathbb{R}_{+} \cup\left\{E_{\mathrm{C} 0,0}(g, \zeta) \leqslant 0\right\}, & \zeta \in\left[\zeta_{g}, \pi / 2\right),\end{cases}
$$

где

$$
\begin{gathered}
\operatorname{tg} \zeta_{g}=\frac{1}{2} \ln \frac{g}{\kappa_{0}}-\psi(1), \\
\partial_{g} \zeta_{g}>0, \quad \zeta_{g} \rightarrow-\frac{\pi}{2} \quad \text { при } \quad g \rightarrow 0, \quad \zeta_{g} \rightarrow \frac{\pi}{2} \quad \text { при } \quad g \rightarrow \infty .
\end{gathered}
$$

Полная система (обобщенных) ортонормированных собственных функций при значениях $\zeta \in\left(-\pi / 2, \zeta_{g}\right)$ состоит из функций

$$
X_{0 \zeta, E_{\mathrm{C}}}(x ; g)=\rho_{\mathrm{C} 0 \zeta}\left(g, E_{\mathrm{C}}\right) C_{\zeta}\left(x ; g, E_{\mathrm{C}}\right), \quad E_{\mathrm{C}} \in \mathbb{R}_{+},
$$

а при $\zeta \in\left[\zeta_{g}, \pi / 2\right)$ - из функций

$$
\begin{aligned}
X_{0 \zeta, E_{\mathrm{C}}}(x ; g) & =\rho_{\mathrm{C} 0 \zeta}\left(g, E_{\mathrm{C}}\right) C_{\zeta}\left(x ; g, E_{\mathrm{C}}\right), \quad E_{\mathrm{C}} \in \mathbb{R}_{+}, \\
X_{0 \zeta, 0}(x ; g) & =Q_{\mathrm{C} 0 \zeta, 0}(g) C_{\zeta}\left(x ; g, E_{\mathrm{C} 0,0}(g, \zeta),\right.
\end{aligned}
$$

где

$$
\begin{gathered}
\rho_{\mathrm{C} 0 \zeta}^{2}\left(g, E_{\mathrm{C}}\right)=\frac{8}{\kappa_{0}} \frac{B_{\mathrm{C}}\left(g, E_{\mathrm{C}}\right)}{4\left(A_{\mathrm{C}}\left(g, E_{\mathrm{C}}\right) \cos \zeta+2 \sin \zeta\right)^{2}+\pi^{2} B_{\mathrm{C}}^{2}(g, E) \cos ^{2} \zeta}, \\
A_{\mathrm{C}}\left(g, E_{\mathrm{C}}\right)=\operatorname{Re} \Omega_{\mathrm{C}}\left(g, E_{\mathrm{C}}\right), \\
B_{\mathrm{C}}\left(g, E_{\mathrm{C}}\right)=\frac{2}{\pi} \operatorname{Im} \Omega_{\mathrm{C}}\left(g, E_{\mathrm{C}}\right)=1-\operatorname{th} \frac{\pi g}{2 \sqrt{E_{\mathrm{C}}}} \\
Q_{\mathrm{C} 0 \zeta, 0}(g)=\frac{1}{\cos \zeta} \sqrt{\frac{2}{\kappa_{0} \partial_{E_{\mathrm{C}}} f_{2, \mathrm{C} \zeta}\left(g, E_{\mathrm{C} 0,0}(g, \zeta)\right)}} .
\end{gathered}
$$

Значения $E_{\mathrm{C} 0,0}(g, \zeta)<0$ являются решениями уравнения

$$
f_{2, \mathrm{C} \zeta}\left(g, E_{\mathrm{C}}\right)=0, \quad f_{2, \mathrm{C} \zeta}\left(g, E_{\mathrm{C}}\right)=\frac{1}{2} \Omega_{\mathrm{C}}\left(g, E_{\mathrm{C}}\right)+\operatorname{tg} \zeta .
$$

При этом

$$
\partial_{E} f_{2 \mathrm{C} \zeta}(g, E)>0, \quad \partial_{g} E_{\mathrm{C} 0,0}(g, \zeta)>0, \quad \partial_{\zeta} E_{\mathrm{C} 0,0}(g, \zeta)<0
$$


В данном случае $f_{2, \mathrm{C}}(g, E)$ как функция от $E$ - гладкая функция в интервале $-\infty<E<0$, монотонно возрастающая от $-\infty$ до значения

$$
\psi(1)-\frac{1}{2} \ln \frac{g}{\kappa_{0}}+\operatorname{tg} \zeta
$$

когда $E$ изменяется от $-\infty$ до -0 . Таким образом, для $\zeta \in\left(-\pi / 2, \zeta_{g}\right)$ уравнение $(32)$ не имеет решений; для любого фиксированного $\zeta \in\left[\zeta_{g}, \pi / 2\right)$ уравнение (32) имеет единственное решение $E_{\mathrm{C} 0,0}(g, \zeta) \in(-\infty, 0]$, монотонно возрастающее от $-\infty$ до 0 , когда $\zeta$ меняется от $\pi / 2-0$ до $\zeta_{g}$. Как функция $g$ при фиксированном $\zeta$ уровень $E_{\mathrm{C} 0,0}(g, \zeta)$ возрастает монотонно от $E_{\mathrm{C} \zeta}+0$, где

$$
E_{\mathrm{C} \zeta}=E_{\mathrm{C} 0,0}(0, \zeta)=-\frac{\kappa_{0}^{2}}{4} e^{4(\operatorname{tg} \zeta-\mathbf{C})-2 \psi(1 / 2)}
$$

до 0 , когда $g$ меняется от +0 до $g_{\zeta}=\kappa_{0} e^{2(\operatorname{tg} \zeta-\mathbf{C})}$.

При $g=0$ имеем

$$
\operatorname{spec} \hat{h}_{\mathrm{C} 0 \zeta}=\mathbb{R}_{+} \cup\left\{E_{\mathrm{C} 0,0}(0, \zeta) \leqslant 0\right\}, \quad \zeta \in(-\pi / 2, \pi / 2),
$$

полная система (обобщенных) ортонормированных собственных функций состоит из функций

$$
\begin{aligned}
X_{0 \zeta, E_{\mathrm{C}}}(x) & =\rho_{\mathrm{C} 0 \zeta}\left(0, E_{\mathrm{C}}\right) C_{\zeta}\left(x ; 0, E_{\mathrm{C}}\right), & E_{\mathrm{C}} & \in \mathbb{R}_{+}, \\
X_{0 \zeta, 0}(x) & =Q_{\mathrm{C} 0 \zeta, 0}(0) C_{\zeta}\left(x ; 0, E_{\mathrm{C} 0,0}(0, \zeta)\right), & \zeta & \in(-\pi / 2, \pi / 2) .
\end{aligned}
$$

При $g<0$ имеем

$$
\operatorname{spec} \hat{h}_{\mathrm{C} 0 \zeta}=\mathbb{R}_{+} \cup\left\{E_{\mathrm{C} 0, n}(g, \zeta)<0, n \in \mathcal{N}_{0}\right\},
$$

где $\mathcal{N}_{0}$ - некоторое подмножество множества целых чисел (см. ниже). Полный набор (обобщенных) ортонормированных собственных функций состоит из функций

$$
\begin{aligned}
X_{0 \zeta, E_{\mathrm{C}}}(x ; g) & =\rho_{\mathrm{C} 0 \zeta}\left(g, E_{\mathrm{C}}\right) C_{\zeta}\left(x ; g, E_{\mathrm{C}}\right), & E_{\mathrm{C}} & \in \mathbb{R}_{+}, \\
X_{0 \zeta, n}(x ; g) & =Q_{\mathrm{C} 0 \zeta, n}(g) C_{\zeta}\left(x ; g, E_{\mathrm{C} 0, n}(g, \zeta)\right), & n & \in \mathcal{N}_{0},
\end{aligned}
$$

где

$$
Q_{\mathrm{C} 0 \zeta, n}=\frac{1}{\cos \zeta} \sqrt{\frac{2}{\left.\kappa_{0} \partial_{E_{\mathrm{C}}} f_{2, \mathrm{C} \zeta}\left(g, E_{\mathrm{C} 0, n}(g, \zeta)\right)\right)}} .
$$

Значения $E_{\mathrm{C} 0, n}(g, \zeta)$ являются решениями уравнения

$$
\begin{gathered}
f_{2, \mathrm{C} \zeta}(g, E)=0, \\
f_{2, \mathrm{C} \zeta}(g, E)=\psi(1)-\frac{1}{2} \psi\left(\frac{1}{2}\right)-\frac{|g|}{2 \sqrt{E}}-\frac{1}{2} \ln \frac{2 \sqrt{E}}{\kappa_{0}}+\operatorname{tg} \zeta, \quad E<0 .
\end{gathered}
$$

Функция $f_{2, \mathrm{C} \zeta}(g, E)$ обладает следующими свойствами:

$$
\partial_{E} f_{2 C \zeta}(g, E)>0,\left.\quad f_{2, \mathrm{C} \zeta}(g, E)\right|_{E=E_{\mathrm{C} 0, n}(g) \pm 0}=\mp \infty, \quad n \in \mathbb{Z}_{+} ;
$$


в каждом интервале $\left(E_{\mathrm{C} 0, n-1}, E_{\mathrm{C} 0, n}\right), n \in \mathbb{Z}_{+}$, где $E_{\mathrm{C} 0-1}=-\infty$, функция $f_{2, \mathrm{C} \zeta}(g, E)$ возрастает монотонно от $-\infty$ до $\infty$, когда значение $E$ меняется от $E_{\mathrm{C} 0, n-1}+0$ до $E_{\mathrm{C} 0, n}-0$; в каждом интервале $\left(E_{\mathrm{C} 0, n-1}, E_{\mathrm{C} 0, n}\right), n \in \mathbb{Z}_{+}$, при любом фиксированном $\zeta \in(-\pi / 2, \pi / 2)$ уравнение (34) имеет единственное решение $E_{\mathrm{C} 0, n}(g, \zeta)$, причем $\partial_{\zeta} E_{\mathrm{C} 0, n}(g, \zeta)<0$, которое монотонно возрастает от $E_{\mathrm{C} 0, n-1}+0$ до $E_{\mathrm{C} 0, n}-0$, когда $\zeta$ меняется от $\pi / 2-0$ до $-\pi / 2+0$. Заметим, что справедливы равенства

$$
\lim _{\zeta \rightarrow-\pi / 2} E_{\mathrm{C} 0, n}(g, \zeta)=\lim _{\zeta \rightarrow \pi / 2} E_{\mathrm{C} 0, n+1}(g, \zeta)=E_{\mathrm{C}, n}(g),
$$

и это означает эквивалентность расширений для $\zeta=-\pi / 2$ и $\zeta=\pi / 2$.

При фиксированном $\zeta$ функции $E_{\mathrm{C} 0, n}(g, \zeta)$ от параметра $g$ ведут себя следующим образом: $\partial_{g} E_{\mathrm{C} 0, n}(g, \zeta)>0$; для всех $n \geqslant 1$ при любом $\zeta$ функции $E_{\mathrm{C} 0, n}(g, \zeta)$ возрастают монотонно от $-\infty$ до -0 , когда $g$ изменяется от $-\infty$ до -0 . Функция $E_{\mathrm{C} 0,0}(g, \zeta)$ возрастает монотонно от $-\infty$ до $E_{\mathrm{C} 0,0}(0, \zeta)-0$ (см. (33)), когда значение $g$ меняется от $-\infty$ до -0 .

Заметим, что функции $E_{\mathrm{C} 0,0}(g, \zeta)$ при $g>0$ и при $g<0$ являются двумя частями одной гладкой кривой, которую мы также обозначим через $E_{\mathrm{C} 0,0}(g, \zeta)$ и которая обладает следующими свойствами: $E_{\mathrm{C} 0,0}(g, \zeta)$ возрастает монотонно от $-\infty$ до 0 , когда $g$ меняется от $-\infty$ до $g_{\zeta}$, пересекая ось $E_{\mathrm{C}}($ т. е. $g=0)$ в точке $E_{\mathrm{C} 0,0}(0, \zeta)$.

\section{4. Сравнение спектров осцилляторных и кулоноподобных двумерных} теорий. Напомним что соответствие между этими двумя теориями осуществляется с помощью преобразований

$$
x=\kappa_{0} u^{2}, \quad E_{\mathrm{C}}=-\frac{\lambda}{4 \kappa_{0}^{2}}, \quad g=-\frac{E_{\mathrm{O}}}{4 \kappa_{0}}, u=\sqrt{\frac{x}{\kappa_{0}}}, \quad E_{\mathrm{O}}=-4 \kappa_{0} g, \quad \lambda=-4 \kappa_{0}^{2} E_{\mathrm{C}} .
$$

Получаем следующее соответствие между параметрами и между функциями осцилляторной и кулоноподобной двумерных теорий:

$$
\begin{gathered}
w_{\mathrm{C}}\left(g, E_{\mathrm{C}}\right)=w_{\mathrm{O}}\left(\lambda, E_{\mathrm{O}}\right), \quad \widetilde{w}_{\mathrm{C}}\left(g, E_{\mathrm{C}}\right)=\widetilde{w}_{\mathrm{O}}\left(\lambda, E_{\mathrm{O}}\right), \quad E_{\mathrm{C}}>0, \lambda<0, \\
\alpha_{\mathrm{C}}\left(g, E_{\mathrm{C}}\right)=\alpha_{\mathrm{O}}\left(\lambda, E_{\mathrm{C}}\right) ;
\end{gathered}
$$

кроме того, при $|m| \geqslant 1$

$$
f_{2, \mathrm{C} m, n}\left(g, E_{\mathrm{C}}\right)=f_{2, \mathrm{O} m, n}\left(\lambda, E_{0}\right), \quad f_{2, \mathrm{C} m, n}\left(g, E_{\mathrm{C} m, n}\right)=0 \Longleftrightarrow f_{2 \mathrm{O} m n}\left(\lambda, E_{\mathrm{O} m, n}\right)=0,
$$

а также

$$
f_{2, \mathrm{C} \zeta}\left(g, E_{\mathrm{C}}\right)=f_{2, \mathrm{O} \zeta}\left(\lambda, E_{\mathrm{O}}\right), \quad f_{2, \mathrm{C} \zeta}\left(g, E_{\mathrm{C} 0, n}(g, \zeta)\right)=0 \Longleftrightarrow f_{2, \mathrm{O} \zeta}\left(\lambda, E_{\mathrm{O} 0, n}(\lambda, \zeta)\right)=0 ;
$$

далее,

$$
\begin{gathered}
E_{\mathrm{C} m, n}\left(g\left(E_{\mathrm{O} m, n}(\lambda)\right)\right)=-\frac{\lambda}{4 \kappa_{0}^{2}}, \quad E_{\mathrm{O} m, n}\left(\lambda\left(E_{\mathrm{C} m, n}(g)\right)\right)=-4 \kappa_{0} g, \quad|m| \geqslant 1, \\
E_{\mathrm{C} 0, n}\left(g\left(E_{\mathrm{O} 0, n}(\lambda, \zeta)\right), \zeta\right)=-\frac{\lambda}{4 \kappa_{0}^{2}}, \quad E_{\mathrm{O} 0, n}\left(\lambda\left(E_{\mathrm{C} 0, n}(g, \zeta)\right), \zeta\right)=-4 \kappa_{0} g, \\
E_{\mathrm{C} \zeta}=-\frac{\kappa_{0}^{4} e^{4(\operatorname{tg} \zeta-\mathrm{C}-2 \psi(1 / 2))}}{4 \kappa_{0}^{2}}=-\frac{\lambda_{\zeta}}{4 \kappa_{0}^{2}},
\end{gathered}
$$




$$
\begin{gathered}
g_{\zeta}=\kappa_{0} e^{2(\operatorname{tg} \zeta-\mathbf{C})}=-\frac{-4 \kappa_{0}^{2} e^{2(\operatorname{tg} \zeta-\mathbf{C})}}{4 \kappa_{0}}=-\frac{E_{\mathrm{O} \zeta}}{4 \kappa_{0}} ; \\
\rho_{\mathrm{C} m}\left(g, E_{\mathrm{C}}\right)=\sqrt{2} \rho_{\mathrm{O} m}\left(\lambda, E_{\mathrm{O}}\right), \quad E_{\mathrm{C}} \geqslant 0, \quad \lambda \leqslant 0, \quad|m| \geqslant 1, \\
\rho_{\mathrm{C} 0 \zeta}\left(g, E_{\mathrm{C}}\right)=\sqrt{2} \rho_{\mathrm{O} 0 \zeta}\left(\lambda, E_{\mathrm{O}}\right) \quad E_{\mathrm{C}} \geqslant 0, \quad \lambda \leqslant 0 ; \\
C_{m}\left(x ; g, E_{\mathrm{C}}\right)=\left(\kappa_{0} x\right)^{1 / 4} O_{m}\left(u ; \lambda, E_{\mathrm{O}}\right), \quad|m| \geqslant 1, \\
C_{k}\left(x ; g, E_{\mathrm{C}}\right)=\left(\kappa_{0} x\right)^{1 / 4} O_{k}\left(u ; \lambda, E_{\mathrm{O}}\right), \quad k=10,20, \zeta ;
\end{gathered}
$$

$$
\begin{aligned}
& X_{m, E_{\mathrm{C}}}(x ; g)=\sqrt{2}\left(\kappa_{0} x\right)^{1 / 4} U_{m, E_{\mathrm{O}}}(u ; \lambda), \quad E_{\mathrm{C}} \geqslant 0, \quad \lambda \leqslant 0, \quad|m| \geqslant 1 \\
& X_{m, n}(x ; g)=\frac{Q_{\mathrm{C} m, n}(g)}{Q_{\mathrm{O} m, n}(\lambda)}\left(\kappa_{0} x\right)^{1 / 4} U_{m, n}(u ; \lambda), \quad E_{\mathrm{C}} \leqslant 0, \quad \lambda \geqslant 0, \quad|m| \geqslant 1, \quad n \in \mathbb{Z}_{+}, \\
& X_{0 \zeta, E_{\mathrm{C}}}(x ; g)=\sqrt{2}\left(\kappa_{0} x\right)^{1 / 4} U_{0 \zeta, E_{\mathrm{O}}}(u ; \lambda), \quad E_{\mathrm{C}} \geqslant 0, \quad \lambda \leqslant 0, \\
& X_{0 \zeta, n}(x ; g)=\frac{Q_{C \zeta, n}(g)}{Q_{\mathrm{O} \zeta, n}(\lambda)}\left(\kappa_{0} x\right)^{1 / 4} U_{0 \zeta, n}(u ; \lambda), \quad E_{\mathrm{C}} \leqslant 0, \quad \lambda \geqslant 0, \quad n \in \mathbb{Z}_{+} .
\end{aligned}
$$

Напомним, что множества функций $\left\{X_{m, E_{\mathrm{C}}}, X_{m, n}\right\}$ и $\left\{X_{0 \zeta, E_{\mathrm{C}}}, X_{0 \zeta, n}\right\}$ образуют полные системы (обобщенных) собственных функций кулоноподобной модели, а множества $\left\{U_{m, E_{\mathrm{O}}}, X_{m, n}\right\}$ и $\left\{U_{0 \zeta, E_{\mathrm{O}}}, U_{0 \zeta, n}\right\}$ - полные системы (обобщенных) собственных функций осцилляторной модели.

На рис. 2 показаны решения соответствующих уравнений для дискретного спектра. Можно видеть полное соответствие решений.

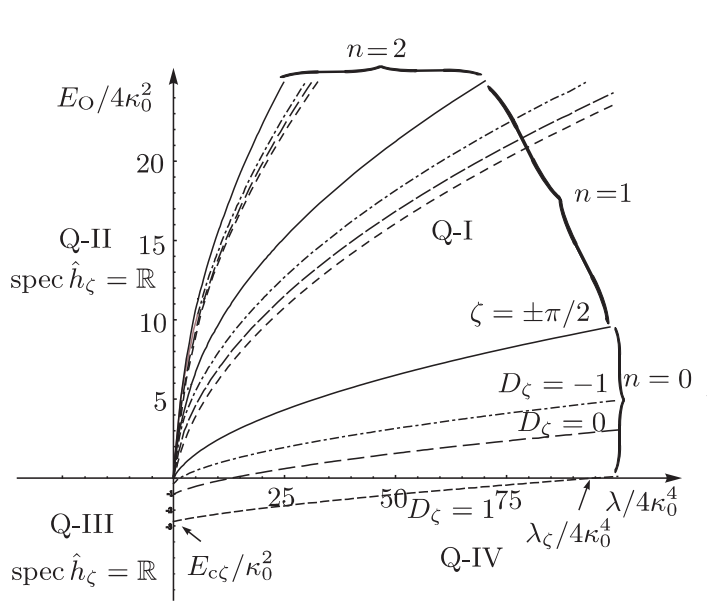

a

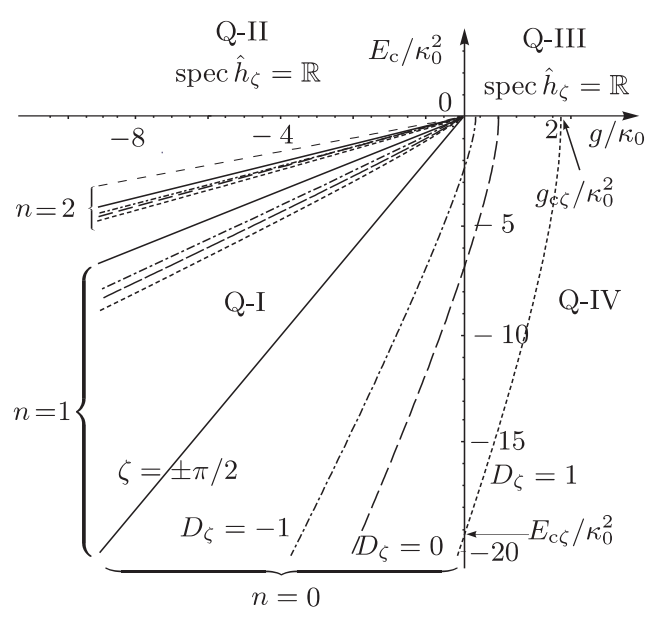

6

Рис. 2. Спектр двумерного осцилляторного (а) и двумерного кулоноподобного (б) потенциалов в случае $m=0$. Соответствующие друг другу квадранты на обоих графиках обозначены одинаково как Q-I, Q-II, Q-III, Q-IV. Здесь $D_{\zeta}=2(\operatorname{tg} \zeta-\mathbf{C})$. 
Соответствие дискретных спектров становится еще более явным, если ввести безразмерные переменные $\mathcal{W}$ и $\mathcal{V}$, которые в случае осциллятора равны

$$
\mathcal{W}=\mathcal{W}_{\mathrm{O}}=\frac{\lambda}{4 \kappa_{0}^{4}}, \quad \mathcal{V}=\mathcal{V}_{\mathrm{O}}=\frac{E_{\mathrm{O}}}{4 \kappa_{0}^{2}},
$$

а в случае кулоноподобного взаимодействия равны

$$
\mathcal{W}=\mathcal{W}_{\mathrm{C}}=-\frac{E_{\mathrm{C}}}{\kappa_{0}^{2}}, \quad \mathcal{V}=\mathcal{V}_{\mathrm{C}}=-\frac{g}{\kappa_{0}} .
$$

Тогда в обоих случаях уравнения (28) и (31) для дискретных спектров в терминах этих переменных совпадают и принимают вид

$$
1+|m|+2 n-\frac{\mathcal{V}}{\sqrt{\mathcal{W}}}=0, \quad \mathcal{W} \geqslant 0 .
$$

Аналогично, в обоих случаях уравнение (29) и уравнение (32) в комбинации с уравнением (34) для дискретных спектров совпадают и принимают вид

$$
2 \mathbf{C}+\ln (2 \sqrt{\mathcal{W}})+\psi\left(\frac{1}{2}-\frac{\mathcal{V}}{2 \sqrt{\mathcal{W}}}\right)=2 \operatorname{tg} \zeta, \quad \mathcal{W} \geqslant 0
$$

\section{4. ЗАКЛЮЧЕНИЕ}

Подведем итоги проведенного рассмотрения. Показано, что между одномерными осцилляторной и кулоноподобной системами, а также между двумерными осцилляторной и кулоноподобной системами существует взаимно однозначное отображение (дуальность) плоскостей константа связи-энергия, $\left(\lambda, E_{\mathrm{O}}\right) \leftrightarrow\left(g, E_{\mathrm{C}}\right)$, и решений соответствующих уравнений Шредингера (3), (4), и уравнений Шредингера (15), (16): $O\left(u ; \lambda, E_{\mathrm{O}}\right) \leftrightarrow C\left(x ; g, E_{\mathrm{C}}\right)$. Это соответствие задается соотношениями (5) в одномерном случае и (17) в двумерном случае.

В двумерном случае для каждого $m \neq 0$ и в случае $m=0$ при фиксированном $\zeta$, а также в одномерном случае при фиксированном $\zeta$ эти отображения обладают следующими свойствами.

1. Каждая точка $\left(\lambda, E_{\mathrm{O}}\right)$-плоскости, являющаяся спектральной ${ }^{1)}$ для осцилляторной задачи, отображается в дуальную точку $\left(g, E_{\mathrm{C}}\right)$-плоскости, являющуюся спектральной для кулоноподобной задачи. При этом спектральные точки $\left(\lambda, E_{\mathrm{O}}\right)$ плоскости, относящиеся к непрерывной, точечной или дискретной частям спектра осцилляторной системы, отображаются в точки $\left(g, E_{\mathrm{C}}\right)$-плоскости, относящиеся соответственно к непрерывной, точечной или дискретной частям спектра кулоноподобной системы. Каждая точка $\left(\lambda, E_{\mathrm{O}}\right)$-плоскости, не являющаяся спектральной для осцилляторной задачи с данной константой связи $\lambda$, отображается в дуальную точку $\left(g, E_{\mathrm{C}}\right)$-плоскости, не являющуюся спектральной для кулоноподобной задачи с соответствующей константой связи $g$ (см. рис. 1,2$)$.

2. Любое решение уравнения Шредингера в случае осцилляторной задачи в точке $\left(\lambda, E_{\mathrm{O}}\right)$, принадлежащее полной ортонормированной системе осцилляторной задачи с данной константой связи $\lambda$, отображается в решение уравнения Шредингера

1) Мы называем точку $\left(\lambda, E_{\mathrm{O}}\right)$-плоскости спектральной, если $E_{\mathrm{O}}$ принадлежит спектру осцилляторной задачи с данной константой связи $\lambda$. Аналогично для случая кулоноподобной системы. 
в случае кулоноподобной задачи в дуальной точке $\left(g, E_{\mathrm{C}}\right)$, принадлежащее полной ортонормированной системе кулоноподобной задачи с дуальной константой связи $g$. При этом волновые функции непрерывной и дискретной частей спектра осцилляторной задачи отображаются соответственно в волновые функции непрерывного и дискретного частей спектра кулоноподобной задачи. Любое решение уравнения Шредингера осцилляторной задачи в точке $\left(\lambda, E_{\mathrm{O}}\right)$, которое не принадлежит полной ортонормированной системе осцилляторной задачи с данной константой связи $\lambda$, отображается в решение уравнения Шредингера кулоноподобной задачи в дуальной точке $\left(g, E_{\mathrm{C}}\right)$, не принадлежащее полной ортонормированной системе кулоноподобной задачи с данной константой связи $g$.

Благодарности. Авторы благодарят А. Нерсесяна за полезные обсуждения рассмотренных вопросов. И.В. Тютин благодарит РФФИ (грант № 11-01-00830) за частичную финансовую поддержку.

\section{Список литературы}

[1] V. Ter-Antonyan, Dyon-oscillator duality, arXiv: quant-ph/0003106.

[2] А.П. Нерсесян, В. М. Тер-Антонян, ЯФ, 61:10 (1998), 1868-1872, arXiv: physics/ 9712027.

[3] Ye. M. Hakobyan, V.M. Ter-Antonyan, ЯФ, 68:10 (2005), 1767-1775, arXiv: quant-ph/ 0002069.

[4] S. Bellucci, A. Nersessian, A. Yeranyan, Phys. Rev. D, 70:4 (2004), 045006, 5 pp., arXiv: hep-th/0312323.

[5] A. Nersessian, G. Pogosian, Phys. Rev. A, 63:2 (2001), 020103, 4 pp., arXiv: quant-ph/0006118.

[6] Л. Г. Мардоян, А. Н. Сисакян, В. М. Тер-Антонян, ЯФ, 61:10 (1998), 1859-1863.

[7] D. Gitman, I. Tyutin, B. Voronov, Self-adjoint Extensions in Quantum Mechanics. General Theory and Applications to Schrödinger and Dirac Equations with Singular Potentials, Progress in Mathematical Physics, 62, Springer, New York, 2012.

[8] I. V. Tyutin, G. V. Grigoryan, R. P. Grigoryan, About dual one-dimensional oscillator and Coulomb-like theories, arXiv: 1011.5359.

[9] M. C. Baldiotti, D. M. Gitman, I. V. Tyutin, B. L. Voronov, Phys. Scripta, 83:6 (2011), 065007, 15 pp.

[10] G. V. Grigoryan, R. P. Grigoryan, I. V. Tyutin, About dual two-dimensional oscillator and Coulomb-like theories on a plane, arXiv: 1112.2209. 